\title{
ROBUST OPTIMAL SWITCHING CONTROL FOR NONLINEAR SYSTEMS*
}

\author{
JOSEPH A. BALL ${ }^{\dagger}$, JERAWAN CHUDOUNG ${ }^{\ddagger}$, AND MARTIN V. DAY ${ }^{\dagger}$
}

\begin{abstract}
We formulate a robust optimal control problem for a general nonlinear system with finitely many admissible control settings and with costs assigned to switching of controls. We formulate the problem both in an $L_{2}$-gain/dissipative system framework and in a game-theoretic framework. We show that, under appropriate assumptions, a continuous switching-storage function is characterized as a viscosity supersolution of the appropriate system of quasi-variational inequalities (the appropriate generalization of the Hamilton-Jacobi-Bellman-Isaacs equation for this context) and that the minimal such switching-storage function is equal to the continuous switching lowervalue function for the game. Finally, we show how a prototypical example with one-dimensional state space can be solved by a direct geometric construction.
\end{abstract}

Key words. running cost, switching cost, worst-case disturbance attenuation, differential game, state-feedback control, nonanticipating strategy, storage function, lower-value function, system of quasi-variational inequalities, viscosity solution

AMS subject classifications. Primary, 49J35; Secondary, 49L20, 49L25, 49J35, 93B36, 93B52

PII. S0363012900372611

1. Introduction. We consider a state-space system $\Sigma_{s w}$

$$
\begin{aligned}
& \dot{y}=f(y, a, b), \\
& z=h(y, a, b),
\end{aligned}
$$

where $y(t) \in \mathbb{R}^{N}$ is the state, $a(t) \in A$ is the control input, $b(t) \in B \subset \mathbb{R}^{M}$ is the deterministic unknown disturbance, and $z(t) \in \mathbb{R}$ is the cost function. We assume that the set $A$ of admissible control values is a finite set, $A=\left\{a^{1}, \ldots, a^{r}\right\}$. The control signals $a(t)$ are then necessarily piecewise constant with values in $A$. We normalize control signals $a(t)$ to be right continuous and refer to the value $a(t)$ as the new current control and $a\left(t^{-}\right)$as the old current control at time $t$. We assume that there is a distinguished input index $i_{0}$ for which $f\left(0, a^{i_{0}}, 0\right)=0$ and $h\left(0, a^{i_{0}}, 0\right)=0$ so that 0 is an equilibrium point for the autonomous system induced by setting $a(t)=a^{i_{0}}$ and $b(t)=0$. In addition, we assume that a cost $k\left(a^{i}, a^{j}\right) \geq 0$ is assigned at each time instant $\tau_{n}$ at which the controller switches from the old current control $a\left(\tau_{n}^{-}\right)=a^{i}$ to the new current control $a\left(\tau_{n}\right)=a^{j}$. For a given old initial control $a\left(0^{-}\right)$, the associated control decision is to choose switching times

$$
0 \leq \tau_{1}<\tau_{2}<\cdots, \quad \lim _{n \rightarrow \infty} \tau_{n}=\infty
$$

and controls

$$
a\left(\tau_{1}\right), a\left(\tau_{2}\right), a\left(\tau_{3}\right), \ldots
$$

*Received by the editors May 26, 2000; accepted for publication (in revised form) February 21, 2002; published electronically September 19, 2002.

http://www.siam.org/journals/sicon/41-3/37261.html

†Department of Mathematics, Virginia Tech, Blacksburg, VA 24061 (ball@math.vt.edu, day@ math.vt.edu).

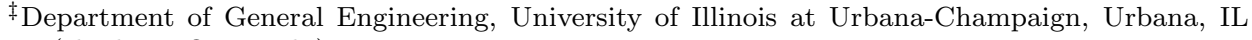
61801 (chudoung@uiuc.edu). 
such that the controller switches from the old current control $a\left(\tau_{n}^{-}\right)$to the new current control $a\left(\tau_{n}\right) \neq a\left(\tau_{n}^{-}\right)$at time $\tau_{n}$, where we set

$$
a(t)= \begin{cases}a\left(0^{-}\right), & t \in\left[0, \tau_{1}\right), \\ a\left(\tau_{n}\right), & t \in\left[\tau_{n}, \tau_{n+1}\right), n=1,2, \ldots,\end{cases}
$$

if $\tau_{1}>0$ and

$$
a(t)=a\left(\tau_{n}\right), \quad t \in\left[\tau_{n}, \tau_{n+1}\right), n=1,2, \ldots,
$$

otherwise. We assume that the state $y(\cdot)$ of (1.1) does not jump at the switching time $\tau_{n}$; i.e., the solution $y(\cdot)$ is assumed to be absolutely continuous. The cost of running the system up to time $T \geq 0$ with initial state $y(0)=x$, old initial control $a\left(0^{-}\right)=a^{j}$, control signal $a$ for $t \geq 0$, and disturbance signal $b$ is given by

$$
C_{T^{-}}\left(x, a^{j}, a, b\right)=\int_{0}^{T} h\left(y_{x}(t, a, b), a(t), b(t)\right) d t+\sum_{\tau: 0 \leq \tau<T} k\left(a\left(\tau^{-}\right), a(\tau)\right) .
$$

We have used the notation $y_{x}(\cdot, a, b)$ for the unique solution of (1.1) corresponding to the choices of the initial condition $y(0)=x$, the control $a(\cdot)$, and the disturbance $b(\cdot)$. In what follows, we will often abbreviate $y_{x}(\cdot, a, b)$ to $y_{x}(\cdot)$ or $y(\cdot)$; the precise meaning should be clear from the context.

As the running cost $h(y(t), a(t), b(t))+k\left(a\left(t^{-}\right), a(t)\right)$, where $a\left(t^{-}\right)=a^{j}$ if $t=0$, involves not only the value $y(t)$ of the state along with the value of the control $a(t)$ and the value of the disturbance $b(t)$ at time $t$ but also the value of the old current control $a\left(t^{-}\right)$, it makes sense to think of the old current control $a\left(t^{-}\right)$at time $t$ as part of an augmented state vector $y^{a u g}(t)=\left(y(t), a\left(t^{-}\right)\right)$at time $t$. This can be done formally by including $a\left(t^{-}\right)$as part of the state vector, in which case a switching control problem becomes an impulse control problem (see [10], where problems of this sort are set in the general framework of hybrid systems). We shall keep the switchingcontrol formalism here; however, in implementing optimization algorithms, we shall see that it is natural to consider augmented state-feedback controls $\left(x, a^{j}\right) \rightarrow a\left(x, a^{j}\right)$ rather than merely state-feedback controls $x \rightarrow a(x)$ in order to obtain solutions. We shall refer to such augmented state-feedback controls $\left(x, a^{j}\right) \rightarrow a\left(x, a^{j}\right) \in A$ as simply switching state-feedback controllers. Note that, while the augmented state is required to compute the instantaneous running cost at time $t$, only the (nonaugmented) state vector $y(t)$ at time $t$ is needed to determine the state trajectory past time $t$ for a given input signal $(a(\cdot), b(\cdot))$ past time $t$.

The precise formulation of our optimal control problem is as follows. First, for a prescribed attenuation level $\gamma>0$ and a given augmented initial state $\left(x, a^{j}\right)$, we seek an admissible control signal $a(\cdot)=a_{x, j}(\cdot)$ with $a\left(0^{-}\right)=a^{j}$ so that

$$
C_{T^{-}}\left(x, a^{j}, a, b\right) \leq \gamma^{2} \int_{0}^{T}|b(t)|^{2} d t+U_{\gamma}^{j}(x)
$$

for all locally $L_{2}$ disturbances $b$, all positive real numbers $T$, and some nonnegativevalued bias function $U_{\gamma}^{j}(x)$ with $U_{\gamma}^{i_{0}}(0)=0$. Note that this inequality corresponds to an input-output system having $L_{2}$-gain at most $\gamma$, where $C_{T^{-}}$replaces the $L_{2^{-}}$ norm of the output signal over the time interval $[0, T]$, and where the equilibrium point is taken to be $\left(0, a^{i_{0}}\right)$ in the augmented state space. The dissipation inequality (1.3) then can be viewed as an $L_{2}$-gain inequality, and our problem can be viewed as 
the analogue of the nonlinear $H^{\infty}$-control problem for systems with switching costs (see [20]). In the switching state-feedback version of the problem, $a(\cdot)$ is a function of the current state and the current old control; i.e., one decides what control to use at time $t$ based on knowledge of the current augmented state $\left(y(t), a\left(t^{-}\right)\right)$. In the standard game-theoretic formulation of the problem, $a(\cdot)$ is a nonanticipating function $a(\cdot)=\alpha_{x}^{j}[b](\cdot)$ (called a strategy) of the disturbance $b$ depending also on the initial state $x$ and initial old control value $a^{j}$; i.e., for a given augmented initial state $\left(x, a^{j}\right)$, the computation of the control value $\alpha_{x}^{j}[b](t)$ at time $t$ uses knowledge only of the past and current values of the disturbance $b(\cdot)$. Second, we ask for the admissible control $a$ with $a\left(0^{-}\right)=a^{j}$ (with whatever information structure) which gives the best system performance in the sense that the nonnegative functions $U_{\gamma}^{j}(x)$ are as small as possible. A closely related problem formulation is to view the switching-control system as a game with payoff function

$$
J_{T^{-}}\left(x, a^{j}, a, b\right)=\int_{[0, T)} l\left(y_{x}(t), a^{j}, a(t), b(t)\right), \quad a\left(0^{-}\right)=a^{j}, j=1, \ldots, r,
$$

where we view $l\left(y_{x}, a^{j}, a, b\right)$ as the measure given by

$$
\begin{aligned}
l\left(y(t), a^{j}, a(t), b(t)\right) & =\left[h(y(t), a(t), b(t))-\gamma^{2}|b(t)|^{2}\right] d t+k\left(a\left(t^{-}\right), a(t)\right) \delta_{t}, \\
\text { with } a\left(0^{-}\right) & =a^{j},
\end{aligned}
$$

where $\delta_{t}$ is the unit point-mass distribution at the point $t$. In this game setting, the disturbance player seeks to use $b(t)$ and $T$ to maximize the payoff, while the control player seeks to use the choice of piecewise-constant right-continuous function $a(t)$ to minimize the payoff. The switching lower value $V_{\gamma}=\left(V_{\gamma}^{1}, \ldots, V_{\gamma}^{r}\right)$ of this game is then given by

$$
V_{\gamma}^{j}(x)=\inf _{\alpha} \sup _{b, T} J_{T^{-}}\left(x, a^{j}, \alpha_{x}^{j}[b], b\right), \quad j=1, \ldots, r,
$$

where the supremum is over all nonnegative real numbers $T$ and all locally $L_{2}$ disturbance signals $b$, while the infimum is over all nonanticipating control strategies $b \rightarrow \alpha_{x}^{j}[b]$ depending on the initial augmented state $\left(x, a^{j}\right)$. By letting $T$ tend to 0 , we see that each component of the switching lower value $V_{\gamma}(x)=\left(V_{\gamma}^{1}(x), \ldots, V_{\gamma}^{r}(x)\right)$ is nonnegative. Then, by construction, $\left(V_{\gamma}^{1}, \ldots, V_{\gamma}^{r}\right)$ gives the smallest possible value which can satisfy (1.3) (with $V_{\gamma}^{j}$ in place of $U_{\gamma}^{j}$ ) for some nonanticipating strategy $\left(x, a^{j}, b\right) \rightarrow \alpha_{x}^{j}[b](\cdot)=a(\cdot)$.

In the standard theory of nonlinear $H^{\infty}$-control, the notion of storage function for a dissipative system plays a prominent role (see [20]). For our setting with switching costs, we say that a nonnegative vector function $S_{\gamma}=\left(S_{\gamma}^{1}, \ldots, S_{\gamma}^{r}\right)$ on $\mathbb{R}^{N}$ is a switching-storage function for the system (1.1)-(1.2) with strategy $\alpha$ if, for all $y(0)=x \in \mathbb{R}^{N}, b$ measurable with values in $B$ and $0 \leq t_{1}<t_{2}$, the following inequality holds:

$$
\begin{aligned}
& S_{\gamma}^{j\left(t_{2}\right)}\left(y_{x}\left(t_{2}, \alpha_{x}^{j}[b], b\right)\right)-S_{\gamma}^{j\left(t_{1}\right)}\left(y_{x}\left(t_{1}, \alpha_{x}^{j}[b], b\right)\right) \\
& \leq \int_{t_{1}}^{t_{2}}\left[\gamma^{2}|b(s)|^{2}-h\left(y_{x}(s), \alpha_{x}^{j}[b](s), b(s)\right)\right] d s \\
& \quad-\sum_{t_{1} \leq \tau<t_{2}} k\left(\alpha_{x}^{j}[b]\left(\tau^{-}\right), \alpha_{x}^{j}[b](\tau)\right)
\end{aligned}
$$


(where $j(t)$ is specified by $\alpha_{x}^{j}[b]\left(t^{-}\right)=a^{j(t)}$ ). The control problem then is to find the switching strategy $\alpha:\left(x, a^{j}, b\right) \rightarrow \alpha_{x}^{j}[b](\cdot)$ which gives the best performance, as measured by obtaining the minimal possible $S_{\gamma}(x)=\left(S_{\gamma}^{1}(x), \ldots, S_{\gamma}^{r}(x)\right)$ as the associated closed-loop switching-storage function. Note that any switching-storage function may serve as the vector bias function $U_{\gamma}=\left(U_{\gamma}^{1}, \ldots, U_{\gamma}^{r}\right)$ in the $L_{2}$-gain inequality (1.3) if, in addition, $S_{\gamma}^{i_{0}}(0)=0$. This suggests that the available switchingstorage function (i.e., the minimal possible switching-storage function over all possible switching strategies) should equal the switching lower-value $V_{\gamma}$ (1.4) for the game described above. We shall see that this is indeed the case with appropriate hypotheses imposed.

Our main results concerning the robust optimal switching-cost problem are as follows: Under minimal smoothness assumptions on the problem data and compactness of the set $B$, the following hold:

(i) $V_{\gamma}^{j}(x) \leq \min _{i \neq j}\left\{V_{\gamma}^{i}(x)+k\left(a^{j}, a^{i}\right)\right\}, x \in \mathbb{R}^{N}, j=1, \ldots, r$.

(ii) If continuous, $V_{\gamma}$ is a viscosity solution in $\mathbb{R}^{N}$ of the system of quasivariational inequalities (SQVI) defined in section 2 (see (2.5)). (The precise definition of viscosity subsolution, supersolution, and solution will be given in section 2.)

(iii) If $S_{\gamma}=\left(S_{\gamma}^{1}, \ldots, S_{\gamma}^{r}\right)$ is a continuous switching-storage function for some strategy $\alpha$, then $S_{\gamma}$ is a nonnegative continuous viscosity supersolution of the SQVI (2.5).

(iv) If $U_{\gamma}=\left(U_{\gamma}^{1}, \ldots, U_{\gamma}^{r}\right)$ is a nonnegative, continuous viscosity supersolution of the SQVI (2.5) and $U_{\gamma}$ has the property (i), then there is a canonical choice of switching state-feedback control strategy $\alpha_{U_{\gamma}}:\left(x, a^{j}, b\right) \rightarrow \alpha_{U_{\gamma}, x}^{j}[b]$ such that $U_{\gamma}$ is a switching-storage function for the closed-loop system formed by using the strategy $\alpha_{U_{\gamma}} ;$ thus

$$
U_{\gamma}^{j}(x) \geq \sup _{b, T}\left\{\int_{[0, T)} l\left(y_{x}(s), a^{j}, \alpha_{U_{\gamma}, x}^{j}[b](s), b(s)\right)\right\} \geq V_{\gamma}^{j}(x) .
$$

The switching lower-value $V_{\gamma}$, if continuous, is characterized as the minimal, nonnegative continuous viscosity supersolution of (2.5) having property (i) above as well as the minimal continuous function satisfying property (i), which is a switching-storage function for the closed-loop system associated with some nonanticipating strategy $\alpha$.

In the precise formulation of our problem, for technical convenience, we impose the condition that the disturbance signals $b(t)$ take values in a bounded subset $B$ of $\mathbb{R}^{M}$; hence our setup technically does not include the linear-quadratic case (where $f$ is linear and $h$ is quadratic). In general, this issue has been a stumbling block for application of the nonlinear dynamic programming formalism to this class of problems. In [21], this difficulty was overcome by an ad hoc reparametrization technique, whereby the general unbounded case was reduced to the bounded case. This would be one approach to removing the boundedness assumption which we have imposed here; however, see also Remark 1 in section 3 below.

The usual formulation of the $H^{\infty}$-control problem also involves a stability constraint. We also prove that, under appropriate conditions, the closed-loop system associated with switching strategy $\alpha_{U_{\gamma}}$ corresponding to the nonnegative continuous supersolution $U_{\gamma}$ of the SQVI is stable. The main idea is to use the supersolution $U_{\gamma}$ as a Lyapunov function for trajectories of the closed-loop system. Related stability problems for systems with control switching are discussed, e.g., by Branicky in [11].

Infinite-horizon optimal switching-control problems are discussed in [6, Chapter III, section 4.4] but with a discount factor in the running cost and no disturbance 
term. Differential games with switching strategies and switching costs for the case of finite horizon problems is discussed in [23], while the case of an infinite horizon with both control and competing disturbance but with a discount factor in the running cost is discussed in [24]. These authors, under their various assumptions, were able to show that the value function is continuous and is the unique solution of the appropriate system of quasi-variational inequalities. However, our formulation has no discount factor in the running cost, so the running cost is not guaranteed to be integrable over the infinite interval $[0, \infty)$. This forces the introduction of the extra "disturbance player" $T$ in (1.4). We establish a dynamic programming principle (DPP) for this setting and derive from it the appropriate system of quasi-variational inequalities (SQVI) to be satisfied by $V_{\gamma}$. While elements of our derivation of the DPP closely follow the known proofs for other cases (see [23], [24]), these proofs do not carry over directly due to a lack of positive discount factor and the presence of the extra disturbance player $T$. Our lower-value function $V_{\gamma}$ probably in general is not continuous and, moreover, cannot be characterized simply as the unique solution of the SQVI as is the case for finite-horizon problems and problems with a positive discount factor. Our formulation of the optimal switching-cost problem is a precise analogue of the standard nonlinear $H^{\infty}$-control problem; our results (particularly the characterization of the switching lower value as the minimal viscosity supersolution of the appropriate SQVI) parallel those of Soravia [21] obtained for the standard nonlinear $H^{\infty}$-control problem (see also [13], [22], and [6, Appendix B] for later, closely related refinements of the nonlinear $H_{\infty}$ results).

Another approach to the derivation of the Hamilton-Jacobi-Bellman-Isaacs (HJBI) equation satisfied by the value function for a differential game is as an application of a comparison principle for the HJBI equation (see [12] or [6]). In [1], this approach was adapted to provide an alternative derivation of the SQVI satisfied by the lower-value function for the robust switching-control problem studied here.

In our companion paper [2], we present a parallel analysis for another analogue of the nonlinear $H^{\infty}$-control problem, namely, a robust stopping-time control problem, where the only control is a decision as to when to stop the system, and there is an instantaneous cost for stopping (dependent on the final state) in addition to the running cost. In this setting, the storage function (or value function if one uses the game interpretation) is a solution of a single variational inequality rather than a coupled system of quasi-variational inequalities as is the case here. The results and general techniques from [2] parallel those of the present paper, but specific details necessarily differ due to the differences in settings. A connection between the two problems is explained in Remark 2 in section 3.

More general types of impulse-control problems have been studied in the literature (see, e.g., [7], [17], [18]) where a general (not necessarily discrete) measure is allowed to enter both the dynamics and the running cost. Such generality leads to a number of complications, such as what is meant by a trajectory of the closed-loop system, how to implement the DPP for discontinuous Hamiltonians, etc. Again, these authors' formulations focus on a finite horizon or assume a discount factor in the running cost. Our purpose here is to work out the details for the switching-control analogue of the standard nonlinear $H^{\infty}$-control problem, where there is an infinite horizon with no discount factor in the running cost for the simpler situation where the singularities in the control are simple jumps.

Original motivation for our work arose from the problem of designing a real-time feedback control for traffic signals at a highway intersection (see [3], [4]), where the 
size of the cost imposed on switching can be used as a tuning parameter to lead to more desirable types of traffic-light signalization. Also, a positive switching cost eliminates the chattering present in the solution otherwise.

The paper is organized as follows. In section 2, we discuss assumptions and definitions. Section 3 presents the main results on the connection between value functions (and storage functions) with systems of quasi-variational inequalities. Section 4 presents stability of the closed-loop switching control system. Finally, section 5 presents an example with one-dimensional state-space, where the value function and associated robust state-feedback control are explicitly computable; a similar example for the setting of the robust stopping-time problem is presented in [1].

2. Preliminaries. Let $A=\left\{a^{1}, a^{2}, \ldots, a^{r}\right\}$ be a finite set, and let $B$ be a compact subset of $\mathbb{R}^{M}$ containing the origin 0 . We consider a general nonlinear system $\Sigma_{s w}$ (see (1.1)-(1.2)) with a switching-cost function $k$. We make the following assumptions on problem data $f, h, k$ :

(A1) $f: \mathbb{R}^{N} \times A \times B \rightarrow \mathbb{R}^{N}$ and $h: \mathbb{R}^{N} \times A \times B \rightarrow \mathbb{R}$ are continuous;

(A2) $f$ and $h$ are bounded on $B(0, R) \times A \times B$ for all $R>0$;

(A3) there are moduli $\omega_{f}$ and $\omega_{h}$ such that

$$
\begin{aligned}
& |f(x, a, b)-f(y, a, b)| \leq \omega_{f}(|x-y|, R), \\
& |h(x, a, b)-h(y, a, b)| \leq \omega_{h}(|x-y|, R)
\end{aligned}
$$

for all $x, y \in B(0, R), R>0, a \in A$, and $b \in B$;

(A4) $(f(x, a, b)-f(y, a, b)) \cdot(x-y) \leq L|x-y|^{2}$ for all $x, y \in \mathbb{R}^{N}, a \in A$, and $b \in B$

(A5) $k: A \times A \rightarrow \mathbb{R}$ and

$$
\begin{aligned}
& k\left(a^{j}, a^{i}\right)<k\left(a^{j}, a^{d}\right)+k\left(a^{d}, a^{i}\right), \\
& k\left(a^{j}, a^{i}\right)>0, \\
& k\left(a^{j}, a^{j}\right)=0
\end{aligned}
$$

for all $a^{d}, a^{i}, a^{j} \in A, d \neq i \neq j$;

(A6) $h(x, a, 0) \geq 0$ for all $x \in \mathbb{R}^{N}, a \in A$.

The set of admissible controls for our problem is the set

$$
\begin{aligned}
\mathcal{A}=\left\{a(\cdot)=\sum_{i \geq 1} a_{i-1} 1_{\left[\tau_{i-1}, \tau_{i}\right)}(\cdot) \mid a_{i} \in A, a_{i} \neq\right. & a_{i-1} \text { for } i \geq 1, \\
& \left.0=\tau_{0} \leq \tau_{1}<\tau_{2}<\cdots, \tau_{i} \uparrow \infty\right\}
\end{aligned}
$$

consisting of piecewise-constant right-continuous functions on $[0, \infty)$ with values in the control set $A$, where we denote by $\tau_{1}, \tau_{2}, \ldots$ the points at which control switchings occur. The set of admissible disturbances is $\mathcal{B}$, which consists of measurable functions on $[0, \infty)$ with values in the set $B$ :

$$
\mathcal{B}=\{b:[0, \infty) \rightarrow B \mid b \text { is measurable on }[0, \infty)\} .
$$

Note that any admissible disturbance $b$ is then locally integrable by the assumption that the disturbance set $B$ is bounded. A strategy is a map $\alpha: \mathbb{R}^{N} \times A \times \mathcal{B} \rightarrow \mathcal{A}$ 
with value at $\left(x, a^{j}, b\right)$ denoted by $\alpha_{x}^{j}[b](\cdot)$. The strategy $\alpha$ assigns control function $a(t)=\alpha_{x}^{j}[b](t)$ if the augmented initial condition is $\left(x, a^{j}\right)$ and the disturbance is $b(\cdot)$. Thus, if it happens that $\tau_{1}>\tau_{0}=0$, then $a(t)=a_{0}=a^{j}$ for $t \in\left[\tau_{0}, \tau_{1}\right)$. Otherwise, $a(t)=a_{1} \neq a^{j}$ for $t \in\left[0, \tau_{2}\right)=\left[\tau_{1}, \tau_{2}\right)$, and an instantaneous charge of $k\left(a^{j}, a(0)\right)$ is incurred at time 0 in the cost function. A strategy $\alpha$ is said to be nonanticipating if, for each $x \in \mathbb{R}^{N}$ and $j \in\{1, \ldots, r\}$, for any $T>0$ and $b, \bar{b} \in \mathcal{B}$, with $b(s)=\bar{b}(s)$ for all $s \leq T$, it follows that $\alpha_{x}^{j}[b](s)=\alpha_{x}^{j}[\bar{b}](s)$ for all $s \leq T$. We denote by $\Gamma$ the set of all nonanticipating strategies:

$$
\begin{aligned}
\Gamma=\left\{\alpha: \mathbb{R}^{N} \times A \times \mathcal{B} \rightarrow\right. & \mathcal{A} \mid \\
& \left.\alpha_{x}^{j} \text { is nonanticipating for each } x \in \mathbb{R}^{N} \text { and } j=1, \ldots, r\right\} .
\end{aligned}
$$

We consider trajectories of the nonlinear system

$$
\left\{\begin{array}{l}
\dot{y}(t)=f(y(t), a(t), b(t)) \\
y(0)=x
\end{array}\right.
$$

Under the assumptions (A1), (A2), and (A4), for given $x \in \mathbb{R}^{N}, a \in \mathcal{A}$, and $b \in \mathcal{B}$, the solution of $(2.1)$ exists uniquely for all $t \geq 0$. We denote by $y_{x}(\cdot, a, b)$ or simply $y_{x}(\cdot)$ the unique solution of (2.1) corresponding to the choice of the initial condition $x \in \mathbb{R}^{N}$, the control $a(\cdot) \in \mathcal{A}$, and the disturbance $b(\cdot) \in \mathcal{B}$. We also have the usual estimates on the trajectories (see, e.g., [6, pp. 97-99]:

$$
\begin{aligned}
\left|y_{x}(t, a, b)-y_{z}(t, a, b)\right| & \leq e^{L t}|x-z|, \quad t>0, \\
\left|y_{x}(t, a, b)-x\right| & \leq M_{x} t, \quad t \in\left[0,1 / M_{x}\right], \\
\left|y_{x}(t, a, b)\right| & \leq(|x|+\sqrt{2 K t}) e^{K t}
\end{aligned}
$$

for all $a \in \mathcal{A}, b \in \mathcal{B}$, where

$$
\begin{aligned}
M_{x} & =\max \{|f(z, a, b)||| x-z \mid \leq 1, a \in A, b \in B\}, \\
K & =L+\max \{|f(0, a, b)| \mid a \in A, b \in B\} .
\end{aligned}
$$

For a specified gain tolerance $\gamma>0$, we define the Hamiltonian function $H^{j}$ : $\mathbb{R}^{N} \times \mathbb{R}^{N} \rightarrow \mathbb{R}$ by setting

$$
H^{j}(y, p)=\min _{b \in B}\left\{-p \cdot f\left(y, a^{j}, b\right)-h\left(y, a^{j}, b\right)+\gamma^{2}|b|^{2}\right\}, \quad j=1, \ldots, r .
$$

Note that $H^{j}(y, p)<+\infty$ for all $y, p \in \mathbb{R}^{N}$ by (A2). Under assumptions (A1)-(A4), one can show that the Hamiltonian $H^{j}$ is continuous on $\mathbb{R}^{N} \times \mathbb{R}^{N}$ and satisfies

$$
\begin{aligned}
& \left|H^{j}(x, p)-H^{j}(y, p)\right| \leq L|x-y||p|+\omega_{h}(|x-y|, R) \\
& \text { for all } p \in \mathbb{R}^{N}, x, y \in B(0, R), R>0, \text { and } \\
& \left|H^{j}(x, p)-H^{j}(x, q)\right| \leq L(|x|+1)|p-q| \text { for all } x, p, q \in \mathbb{R}^{N} .
\end{aligned}
$$

We now introduce the system of quasi-variational inequalities (SQVI): for $j=$ $1,2, \ldots, r$

$$
\max \left\{H^{j}\left(x, D u^{j}(x)\right), u^{j}(x)-\min _{i \neq j}\left\{u^{i}(x)+k\left(a^{j}, a^{i}\right)\right\}\right\}=0, \quad x \in \mathbb{R}^{N} .
$$


Definition 1. A vector function $u=\left(u^{1}, u^{2}, \ldots, u^{r}\right)$, where $u^{j} \in C\left(\mathbb{R}^{N}\right)$, is a viscosity subsolution of the SQVI (2.5) if, for any $\varphi^{j} \in C^{1}\left(\mathbb{R}^{N}\right)$,

$$
\max \left\{H^{j}\left(x_{0}, D \varphi^{j}\left(x_{0}\right)\right), u^{j}\left(x_{0}\right)-\min _{i \neq j}\left\{u^{i}\left(x_{0}\right)+k\left(a^{j}, a^{i}\right)\right\}\right\} \leq 0, \quad j=1,2, \ldots, r
$$

at any local maximum point $x_{0} \in \mathbb{R}^{N}$ of $u^{j}-\varphi^{j}$. Similarly, $u$ is a viscosity supersolution of the $S Q V I(2.5)$ if, for any $\varphi^{j} \in C^{1}\left(\mathbb{R}^{N}\right)$,

$$
\max \left\{H^{j}\left(x_{1}, D \varphi^{j}\left(x_{1}\right)\right), u^{j}\left(x_{1}\right)-\min _{i \neq j}\left\{u^{i}\left(x_{1}\right)+k\left(a^{j}, a^{i}\right)\right\}\right\} \geq 0, \quad j=1,2, \ldots, r,
$$

at any local minimum point $x_{1} \in \mathbb{R}^{N}$ of $u^{j}-\varphi^{j}$. Finally, $u$ is a viscosity solution of the SQVI (2.5) if it is simultaneously a viscosity sub- and supersolution.

3. Main results. In this section, we show the connection of the lower value function $V_{\gamma}=\left(V_{\gamma}^{1}, \ldots, V_{\gamma}^{r}\right)$ (see (1.4)) (and a switching-storage function) with the SQVI (2.5).

We begin with the application of the DPP to this setting and then derive some properties of the lower-value vector function $V_{\gamma}$ (see (1.4)). We then use these properties to show that $V_{\gamma}$, if continuous, is a viscosity solution of the SQVI (2.5). Throughout this section, we assume that $V_{\gamma}$ is finite.

Proposition 3.1. Assume (A1)-(A5). Then, for $j=1,2, \ldots, r$ and $x \in \mathbb{R}^{N}$, the lower-value vector function $V_{\gamma}=\left(V_{\gamma}^{1}, \ldots, V_{\gamma}^{r}\right)$ given by (1.4) satisfies

$$
V_{\gamma}^{j}(x) \leq \min _{i \neq j}\left\{V_{\gamma}^{i}(x)+k\left(a^{j}, a^{i}\right)\right\}
$$

Proof. Fix a pair of indices $i, j \in\{1, \ldots, r\}$ with $i \neq j$. For a given $x \in \mathbb{R}^{n}$, $\alpha \in \Gamma, b \in \mathcal{B}$, and $T>0$, we have

$$
\begin{aligned}
& \int_{[0, T)} l\left(y_{x}(s), a^{j}, \alpha_{x}^{j}[b](x), b(s)\right) \\
&=k\left(a^{j}, \alpha_{x}^{j}[b](0)\right)+\int_{[0, T)} l\left(y_{x}(s), \alpha_{x}^{j}[b](0), \alpha_{x}^{j}[b](s), b(s)\right) .
\end{aligned}
$$

Note that there are three cases to consider: (i) $\alpha_{x}^{j}[b](0)=j$, (ii) $\alpha_{x}^{j}[b](0)=i$, (iii) $\alpha_{x}^{j}[b](0) \neq j \neq i$. If (i) or (ii) occurs, then

$$
\begin{aligned}
\int_{[0, T)} l\left(y_{x}(s), a^{j}, \alpha_{x}^{j}[b](x), b(s)\right) \\
\quad<k\left(a^{j}, a^{i}\right)+k\left(a^{i}, \alpha_{x}^{j}[b](0)\right)+\int_{[0, T)} l\left(y_{x}(s), \alpha_{x}^{j}[b](0), \alpha_{x}^{j}[b](s), b(s)\right) \\
\quad=k\left(a^{j}, a^{i}\right)+\int_{[0, T)} l\left(y_{x}(s), a^{i}, \alpha_{x}^{j}[b](s), b(s)\right) .
\end{aligned}
$$


If (iii) occurs, then

$$
\begin{aligned}
\int_{[0, T)} l\left(y_{x}(s), a^{j}, \alpha_{x}^{j}[b](x), b(s)\right) \\
=k\left(a^{j}, \alpha_{x}^{j}[b](0)\right)-k\left(a^{i}, \alpha_{x}^{j}[b](0)\right) \\
\quad+k\left(a^{i}, \alpha_{x}^{j}[b](0)\right)+\int_{[0, T)} l\left(y_{x}(s), \alpha_{x}^{j}[b](0), \alpha_{x}^{j}[b](s), b(s)\right) \\
\quad k\left(a^{j}, \alpha_{x}^{j}[b](0)\right)-k\left(a^{i}, \alpha_{x}^{j}[b](0)\right)+\int_{[0, T)} l\left(y_{x}(s), a^{i}, \alpha_{x}^{j}[b](s), b(s)\right) \\
<k\left(a^{j}, a^{i}\right)+\int_{[0, T)} l\left(y_{x}(s), a^{i}, \alpha_{x}^{j}[b](s), b(s)\right),
\end{aligned}
$$

where the last inequality follows from (A5). By the definition of $V_{\gamma}^{j}(x)$, we have

$$
V_{\gamma}^{j}(x) \leq \sup _{b \in \mathcal{B}, T \geq 0} \int_{[0, T)} l\left(y_{x}(s), a^{j}, \alpha_{x}^{j}[b](s), b(s)\right)
$$

for all $\alpha \in \Gamma$. Taking the supremum over $b \in \mathcal{B}$ and $T \geq 0$ on the right-hand side of (3.1) or (3.2) therefore gives

$$
V_{\gamma}^{j}(x) \leq k\left(a^{j}, a^{i}\right)+\sup _{b \in \mathcal{B}, T \geq 0} \int_{[0, T)} l\left(y_{x}(s), a^{i}, \alpha_{x}^{j}[b](s), b(s)\right) .
$$

Given any strategy $\alpha \in \Gamma$, we can always find another $\widetilde{\alpha} \in \Gamma$ with $\widetilde{\alpha}_{x}^{i}[b]=\alpha_{x}^{j}[b]$ for each $b \in \mathcal{B}$, and, conversely, for any $\widetilde{\alpha} \in \Gamma$, there is an $\alpha \in \Gamma$ so that $\widetilde{\alpha}_{x}^{i}$ is determined by $\alpha$ in this way. Hence, taking the infimum over all $\alpha \in \Gamma$ in the last terms on the right-hand side of (3.3) leaves us with $V_{\gamma}^{i}(x)$. Thus

$$
V_{\gamma}^{j}(x) \leq k\left(a^{j}, a^{i}\right)+V_{\gamma}^{i}(x) .
$$

Since $i \neq j$ is arbitrary, the result follows.

TheOrem 3.2 (DPP). Assume (A1)-(A4). Then, for $j=1,2, \ldots, r, t>0$, and $x \in \mathbb{R}^{N}$, we have

$$
\begin{aligned}
V_{\gamma}^{j}(x)=\inf _{\alpha \in \Gamma} \sup _{b \in \mathcal{B}, T>0}\{ & \int_{[0, t \wedge T)} l\left(y_{x}\left(s, a^{j}, \alpha_{x}^{j}[b], b\right), \alpha_{x}^{j}[b](s), b(s)\right) \\
& \left.+1_{[0, T)}(t) V_{\gamma}^{i}\left(y_{x}\left(t, \alpha_{x}^{j}[b], b\right)\right) \text { such that } \alpha_{x}^{j}[b]\left(t^{-}\right)=a^{i}\right\}
\end{aligned}
$$

where

$$
l\left(y(s), a^{j}, a(s), b(s)\right)=\left[h(y(s), a(s), b(s))-\gamma^{2}|b(s)|^{2}\right] d s+k\left(a\left(s^{-}\right), a(s)\right) \delta_{s}
$$

with $a\left(0^{-}\right)=a^{j}$.

Proof. Fix $x \in \mathbb{R}^{N}, j \in\{1,2, \ldots, r\}$, and $t>0$. We denote by $\omega(x)$ the righthand side of (3.4). Let $\epsilon>0$. For any $z \in \mathbb{R}^{N}$ and any $a^{\ell} \in A$, we pick $\bar{\alpha} \in \Gamma$ such that

$$
V_{\gamma}^{\ell}(z)+\epsilon \geq \int_{[0, T)} l\left(y_{z}(s), a^{\ell}, \bar{\alpha}_{z}^{\ell}[b](s), b(s)\right) \quad \text { for all } b \in \mathcal{B}, \text { for all } T>0
$$


We first want to show that $\omega(x) \geq V_{\gamma}^{j}(x)$. Choose $\hat{\alpha} \in \Gamma$ such that

$$
\begin{aligned}
\omega(x)+\epsilon \geq \sup _{b \in \mathcal{B}, T \geq 0}\left\{\int _ { [ 0 , t \wedge T ) } l \left(y_{x}(s),\right.\right. & \left.a^{j}, \hat{\alpha}_{x}^{j}[b](s), b(s)\right) \\
& \left.+1_{[0, T)}(t) V_{\gamma}^{i}\left(y_{x}(t)\right), \hat{\alpha}_{x}^{j}[b]\left(t^{-}\right)=a^{i}\right\} .
\end{aligned}
$$

For each $b \in \mathcal{B}$ and $T>0$, choose $\delta \in \Gamma$ so that

$$
\delta_{x}^{j}[b](s)= \begin{cases}\hat{\alpha}_{x}^{j}[b](s), & s<t \wedge T \\ \bar{\alpha}_{z}^{i}[b(\cdot+t \wedge T)](s-(t \wedge T)), & s \geq t \wedge T\end{cases}
$$

with $z=y_{x}\left(t \wedge T, \hat{\alpha}_{x}^{j}[b], b\right)$ and $a^{i}=\hat{\alpha}_{x}^{j}[b](t \wedge T)$. Clearly, $\delta_{x}^{j}$ is nonanticipating because $\hat{\alpha}_{x}^{j}$ and $\bar{\alpha}_{z}^{i}$ are. Note that

$$
y_{x}\left(s+t \wedge T, \delta_{x}^{j}[b], b\right)=y_{z}\left(s, \bar{\alpha}_{z}^{i}[b(\cdot+t \wedge T)], b(\cdot+t \wedge T)\right) \text { for } s \geq 0 .
$$

Thus, by the change of variables $\tau=s+t \wedge T$, we have

$$
\begin{aligned}
\int_{[0, T-(t \wedge T))} l\left(y_{z}(s), a^{i}, \bar{\alpha}_{z}^{i}[b(\cdot+t \wedge T)](s),\right. & b(s+t \wedge T)) \\
= & \int_{[t \wedge T, T)} l\left(y_{x}(\tau), a^{j}, \delta_{x}^{j}[b](\tau), b(\tau)\right) .
\end{aligned}
$$

As a consequence of (3.5), (3.6), and (3.7), we have

$$
\begin{aligned}
2 \omega(x)+2 \epsilon \geq & \sup _{b \in \mathcal{B}, T>0}\left\{\int_{[0, t \wedge T)} l\left(y_{x}(s), a^{j}, \hat{\alpha}_{x}^{j}[b](s), b(s)\right)\right. \\
& \left.+1_{[0, T)}(t) \int_{[t \wedge T, T)} l\left(y_{z}(s), a^{i}, \bar{\alpha}_{z}^{i}[b](s), b(s)\right)\right\} \\
= & \sup _{b \in \mathcal{B}, T>0}\left\{\int_{[0, T)} l\left(y_{x}(s), a^{j}, \delta_{x}^{j}[b](s), b(s)\right)\right\} \\
\geq & \inf _{\alpha \in \Gamma} \sup _{b \in \mathcal{B}, T>0}\left\{\int_{[0, T)} l\left(y_{x}(s), a^{j}, \alpha_{x}^{j}[b](s), b(s)\right)\right\} \\
= & V_{\gamma}^{j}(x) .
\end{aligned}
$$

Since $\epsilon>0$ is arbitrary, we conclude that $\omega(x) \geq V_{\gamma}^{j}(x)$.

Next we want to show that $\omega(x) \leq V_{\gamma}^{j}(x)$. From the definition of $\omega(x)$, choose $b_{1} \in \mathcal{B}$ and $T_{1} \geq 0$ such that

$$
\omega(x)-\epsilon \leq \int_{\left[0, T_{1} \wedge t\right)} l\left(y_{x}(s), a^{j}, \bar{\alpha}_{x}^{j}\left[b_{1}\right](s), b_{1}(s)\right)+1_{\left[0, T_{1}\right)}(t) V_{\gamma}^{i}\left(y_{x}(t)\right),
$$

where $\bar{\alpha}_{x}^{j}$ is defined as in (3.5) and $\bar{\alpha}_{x}^{j}\left[b_{1}\right]\left(t^{-}\right)=a^{i}$ for some $a^{i} \in A$. If $t \geq T_{1}$, we 
have

$$
\begin{aligned}
\omega(x)-\epsilon & \leq \int_{\left[0, T_{1}\right)} l\left(y_{x}(s), a^{j}, \bar{\alpha}_{x}^{j}\left[b_{1}\right](s), b_{1}(s)\right) \\
& \leq \sup _{b \in \mathcal{B}, T>0}\left\{\int_{[0, T)} l\left(y_{x}(s), a^{j}, \bar{\alpha}_{x}^{j}[b](s), b(s)\right)\right\} \\
& \leq V_{\gamma}^{j}(x)+\epsilon,
\end{aligned}
$$

where the last inequality follows from (3.5). If $t<T_{1}$, we have

$$
\omega(x)-\epsilon \leq \int_{[0, t)} l\left(y_{x}(s), \bar{\alpha}_{x}^{j}\left[b_{1}\right](s), b_{1}(s)\right)+V_{\gamma}^{i}\left(y_{x}(t)\right) .
$$

Set $z=y_{x}\left(t, \bar{\alpha}_{x}^{j}\left[b_{1}\right], b_{1}\right)$. For each $b \in \mathcal{B}$, define $\tilde{b} \in \mathcal{B}$ by

$$
\tilde{b}(s)= \begin{cases}b_{1}(s), & s<t, \\ b(s-t), & s \geq t,\end{cases}
$$

and choose $\widehat{\alpha} \in \Gamma$ so that

$$
\widehat{\alpha}_{z}[b](s)=\bar{\alpha}_{x}^{j}[\tilde{b}](s+t) \quad \text { for } s \geq 0 .
$$

By definition of $V_{\gamma}^{i}$, choose $b_{2} \in \mathcal{B}$ and $T_{2}>0$ such that

$$
V_{\gamma}^{i}(z)-\epsilon \leq \int_{\left[0, T_{2}\right)} l\left(y_{z}(s), a^{i}, \widehat{\alpha}_{z}\left[b_{2}\right](s), b_{2}(s)\right) .
$$

Then, by change of variable $\tau=s+t$, we have

$$
V_{\gamma}^{i}(z)-\epsilon \leq \int_{\left[t, t+T_{2}\right)} l\left(y_{x}(\tau), a^{j}, \bar{\alpha}_{x}^{j}\left[\tilde{b}_{2}\right](\tau), \tilde{b}_{2}(\tau)\right) .
$$

As a consequence of (3.9) and (3.10), we have

$$
\begin{aligned}
\omega(x)-2 \epsilon & \leq \int_{[0, t)} l\left(y_{x}(s), a^{j}, \bar{\alpha}_{x}^{j}\left[b_{1}\right](s), b_{1}(s)\right)+\int_{\left[t, t+T_{2}\right)} l\left(y_{x}(\tau), a^{j}, \bar{\alpha}_{x}^{j}\left[\tilde{b}_{2}\right](\tau), \tilde{b}_{2}(\tau)\right) \\
& =\int_{\left[0, t+T_{2}\right)} l\left(y_{x}(\tau), a^{j}, \bar{\alpha}_{x}^{j}\left[\tilde{b}_{2}\right](\tau), \tilde{b}_{2}(\tau)\right) \\
& \leq \sup _{b \in \mathcal{B}, T>0}\left\{\int_{[0, T)} l\left(y_{x}(\tau), a^{j}, \bar{\alpha}_{x}^{j}[b](\tau), b(\tau)\right)\right\} \\
& \leq V_{\gamma}^{j}(x)+\epsilon
\end{aligned}
$$

where the last inequality follows from (3.5). Since $\epsilon>0$ is arbitrary, for both cases we have $\omega(x) \leq V_{\gamma}^{j}(x)$ as required.

Corollary 3.3. Assume (A1)-(A4) and (A6). Then, for each $j \in\{1, \ldots, r\}$, $x \in \mathbb{R}^{N}$, and $t>0$, we have

$$
\begin{aligned}
V_{\gamma}^{j}(x) & \leq \sup _{b \in \mathcal{B}, T>0}\left\{\int_{0}^{t \wedge T}\left[h\left(y_{x}(s), a^{j}, b(s)\right)-\gamma^{2}|b(s)|^{2}\right] d s+1_{[0, T)}(t) V_{\gamma}^{j}\left(y_{x}(t)\right)\right\} \\
& \leq \sup _{b \in \mathcal{B}} \int_{0}^{t}\left[h\left(y_{x}(s), a^{j}, b(s)\right)-\gamma^{2}|b(s)|^{2}\right] d s+V_{\gamma}^{j}\left(y_{x}(t)\right) .
\end{aligned}
$$


Proof. Fix $j \in\{1, \ldots, r\}, x \in \mathbb{R}^{N}$, and $t>0$. Define $\alpha \in \Gamma$ by setting $\alpha_{x}^{j}[b](s)=$ $a^{j}$ for all $s \geq 0$ for each $b \in \mathcal{B}$. By Theorem 3.2, we have

$$
V_{\gamma}^{j}(x) \leq \sup _{b \in \mathcal{B}, T>0}\left\{\int_{0}^{t \wedge T}\left[h\left(y_{x}(s), a^{j}, b(s)\right)-\gamma^{2}|b(s)|^{2}\right] d s+1_{[0, T)}(t) V_{\gamma}^{j}\left(y_{x}(t)\right)\right\},
$$

and (3.11) follows.

To prove the second inequality (3.12), consider any $b \in \mathcal{B}$ and $T$ with $0<T<t$. Define a new $\bar{b} \in \mathcal{B}$ by $\bar{b}(s)=b(s)$ for $s \leq T$ and $\bar{b}(s)=0$ for $s>T$. It follows that $y_{x}\left(s, a^{j}, b\right)=y_{x}\left(s, a^{j}, \bar{b}\right)$ for $0 \leq s \leq T$. For $s>T$, we have

$$
h\left(y_{x}(s), a^{j}, b(s)\right)-\gamma^{2}|b(s)|^{2}=h\left(y_{x}(s), a^{j}, 0\right) \geq 0
$$

(by (A6)). Since we also know that $V_{\gamma}^{j}(x) \geq 0$ for all $x \in \mathbb{R}^{N}$ (take $T=0$ in the definition (1.4)), we get

$$
\begin{aligned}
& \int_{0}^{t \wedge T}\left[h\left(y_{x}(s), a^{j}, b(s)\right)-\gamma^{2}|b(s)|^{2}\right] d s+1_{[0, T)}(t) V_{\gamma}^{j}\left(y_{x}(t)\right) \\
& \leq \int_{0}^{t}\left[h\left(y_{x}(s), a^{j}, \bar{b}(s)\right)-\gamma^{2}|b(s)|^{2}\right] d s+V_{\gamma}^{j}\left(y_{x}\left(t, a^{j}, \bar{b}\right)\right),
\end{aligned}
$$

and (3.12) follows as well.

Proposition 3.4. Assume (A1)-(A5). Suppose that, for each $j \in\{1, \ldots, r\}, V^{j}$ is continuous. If $V_{\gamma}^{j}(x)<\min _{i \neq j}\left\{V_{\gamma}^{i}(x)+k\left(a^{j}, a^{i}\right)\right\}$, then there exists $\tau=\tau_{x}>0$ such that, for $0<t<\tau_{x}$,

$$
V_{\gamma}^{j}(x)=\sup _{b \in \mathcal{B}, T>0}\left\{\int_{0}^{t \wedge T}\left[h\left(y_{x}(s), a^{j}, b(s)\right)-\gamma^{2}|b(s)|^{2}\right] d s+1_{[0, T)}(t) V_{\gamma}^{j}\left(y_{x}(t)\right)\right\} .
$$

Proof. We assume $V_{\gamma}^{j}(x)<\min _{i \neq j}\left\{V_{\gamma}^{i}(x)+k\left(a^{j}, a^{i}\right)\right\}$. From Corollary 3.3, we know that, for all $t>0$,

$$
V_{\gamma}^{j}(x) \leq \sup _{b \in \mathcal{B}, T>0}\left\{\int_{0}^{t \wedge T}\left[h\left(y_{x}(s), a^{j}, b(s)\right)-\gamma^{2}|b(s)|^{2}\right] d s+1_{[0, T)}(t) V_{\gamma}^{j}\left(y_{x}(t)\right)\right\} .
$$

Suppose there is a sequence $\left\{t_{n}\right\}$ with $0<t_{n}<\frac{1}{n}$ for $n=1,2, \ldots$ such that

$$
V_{\gamma}^{j}(x)<\sup _{b \in \mathcal{B}, T>0}\left\{\int_{0}^{t_{n} \wedge T}\left[h\left(y_{x}(s), a^{j}, b(s)\right)-\gamma^{2}|b(s)|^{2}\right] d s+1_{[0, T)}\left(t_{n}\right) V_{\gamma}^{j}\left(y_{x}\left(t_{n}\right)\right)\right\} .
$$

Let $w\left(x, t_{n}\right)$ be the right-hand side of (3.13). For each $t_{n}$, define $\epsilon_{n}=\frac{1}{3}\left[w\left(x, t_{n}\right)-\right.$ $\left.V_{\gamma}^{j}(x)\right]$. As $t_{n} \rightarrow 0$ as $n \rightarrow \infty$, from (3.13) we see that $w\left(x, t_{n}\right) \rightarrow V_{\gamma}^{j}(x)$, and hence $\epsilon_{n} \rightarrow 0$ as $n \rightarrow \infty$. It follows that

$$
V_{\gamma}^{j}(x)+\epsilon_{n}<w\left(x, t_{n}\right)-\epsilon_{n} .
$$

Choose $b_{n} \in \mathcal{B}$ and $T_{n} \geq 0$ such that

$$
w\left(x, t_{n}\right)-\epsilon_{n} \leq \int_{0}^{t_{n} \wedge T_{n}}\left[h\left(y_{x}(s), a^{j}, b_{n}(s)\right)-\gamma^{2}\left|b_{n}(s)\right|^{2}\right] d s+1_{\left[0, T_{n}\right)}\left(t_{n}\right) V_{\gamma}^{j}\left(y_{x}\left(t_{n}\right)\right) .
$$


By Theorem 3.2, choose $\alpha_{n} \in \Gamma$ such that

$$
V_{\gamma}^{j}(x)+\epsilon_{n} \geq \int_{\left[0, t_{n} \wedge T_{n}\right]} l\left(y_{x}(s), a^{j},\left(\alpha_{n}\right)_{x}^{j}\left[b_{n}\right](s), b_{n}(s)\right)+1_{\left[0, T_{n}\right)}\left(t_{n}\right) V_{\gamma}^{i_{n}}\left(y_{x}\left(t_{n}\right)\right),
$$

where $\left(\alpha_{n}\right)_{x}^{j}\left[b_{n}\right]\left(t_{n}^{-}\right)=a^{i_{n}} \in A$. From (3.14), (3.15), and (3.16), we have

$$
\begin{aligned}
& \int_{\left[0, t_{n} \wedge T_{n}\right)} l\left(y_{x}(s), a^{j},\left(\alpha_{n}\right)_{x}^{j}\left[b_{n}\right](s), b_{n}(s)\right)+1_{\left[0, T_{n}\right)}\left(t_{n}\right) V_{\gamma}^{i_{n}}\left(y_{x}\left(t_{n}\right)\right) \\
& <\int_{0}^{t_{n} \wedge T_{n}}\left[h\left(y_{x}(s), a^{j}, b_{n}(s)\right)-\gamma^{2}\left|b_{n}(s)\right|^{2}\right] d s+1_{\left[0, T_{n}\right)}\left(t_{n}\right) V_{\gamma}^{j}\left(y_{x}\left(t_{n}\right)\right) .
\end{aligned}
$$

This implies that $\left(\alpha_{n}\right)_{x}^{j}\left[b_{n}\right]$ jumps in the interval $\left[0, t_{n} \wedge T_{n}\right]$. Without loss of generality, assume the number of switchings is equal to $d_{n}$. If $t_{n}<T_{n}$ for infinitely many $n$, by going down to a subsequence we may assume that $t_{n} \leq T_{n}$ for all $n$. From (3.16), we have

$$
\begin{aligned}
V_{\gamma}^{j}(x) \geq \limsup _{n \rightarrow \infty}\left\{\int_{\left[0, t_{n} \wedge T_{n}\right)} l\left(y_{x}(s), a^{j}, \alpha_{x, n}^{j}\left[b_{n}\right](s), b_{n}(s)\right)\right. \\
\left.+1_{\left[0, T_{n}\right)}\left(t_{n}\right) V_{\gamma}^{i_{n}}\left(y_{x}\left(t_{n}\right)\right), \alpha_{x, n}^{j}\left[b_{n}\right]\left(t_{n}^{-}\right)=a^{i_{n}} \in A\right\} \\
=\limsup _{n \rightarrow \infty}\left\{\int_{0}^{t_{n}}\left[h\left(y_{x}(s), \alpha_{x, n}^{j}\left[b_{n}\right](s), b_{n}(s)\right)-\gamma^{2}\left|b_{n}(s)\right|^{2}\right] d s\right. \\
\left.+\sum_{m=1}^{d_{n}} k\left(a_{m-1}, a_{m}\right)+V_{\gamma}^{i_{n}}\left(y_{x}\left(t_{n}\right)\right), \alpha_{x, n}^{j}\left[b_{n}\right]\left(t_{n}\right)=a^{i_{n}} \in A\right\} \\
=\limsup _{n \rightarrow \infty}\left\{\sum_{m=1}^{d_{n}} k\left(a_{m-1}, a_{m}\right)+V_{\gamma}^{i_{n}}\left(y_{x}\left(t_{n}\right)\right), \alpha_{x, n}^{j}\left[b_{n}\right]\left(t_{n}^{-}\right)=a^{i_{n}} \in A\right\} .
\end{aligned}
$$

By using the continuity of $V_{\gamma}^{i_{n}}$ and $\sum_{m=1}^{d_{n}} k\left(a_{m-1}, a_{m}\right)>k\left(a^{j}, a^{i_{n}}\right)$, we have

$$
V_{\gamma}^{j}(x) \geq \min _{i \neq j}\left\{V_{\gamma}^{i}(x)+k\left(a^{j}, a^{i}\right)\right\}
$$

which contradicts one of the assumptions. If $t_{n} \geq T_{n}$ for infinitely many $n$, again without loss of generality we may assume that $t_{n} \geq T_{n}$ for all $n$. From (3.17), we have

$$
\begin{aligned}
& \liminf _{n \rightarrow \infty}\left\{\int_{\left[0, T_{n}\right]} l\left(y_{x}(s), a^{j} \alpha_{x, n}^{j}\left[b_{n}\right](s), b_{n}(s)\right)\right\} \\
& \leq \limsup _{n \rightarrow \infty}\left\{\int_{0}^{T_{n}}\left[h\left(y_{x}(s), a^{j}, b_{n}(s)\right)-\gamma^{2}\left|b_{n}(s)\right|^{2}\right] d s\right\},
\end{aligned}
$$

or, equivalently,

$$
\begin{aligned}
& \liminf _{n \rightarrow \infty}\left\{\int_{0}^{T_{n}}\left[h\left(y_{x}(s), \alpha_{x, n}^{j}\left[b_{n}\right](s), b_{n}(s)\right)-\gamma^{2}\left|b_{n}(s)\right|^{2}\right] d s+\sum_{m=1}^{d_{n}} k\left(a_{m-1}, a_{m}\right)\right\} \\
& \leq \limsup _{n \rightarrow \infty}\left\{\int_{0}^{T_{n}}\left[h\left(y_{x}(s), a^{j}, b_{n}(s)\right)-\gamma^{2}\left|b_{n}(s)\right|^{2}\right] d s\right\} .
\end{aligned}
$$


Thus

$$
\begin{aligned}
\liminf _{n \rightarrow \infty}\left\{\sum_{m=1}^{d_{n}} k\left(a_{m-1}, a_{m}\right)\right\} \leq & \limsup _{n \rightarrow \infty}\left\{\int_{0}^{T_{n}} h\left(y_{x}(s), a^{j}, b_{n}(s)\right) d s\right\} \\
& -\liminf _{n \rightarrow \infty}\left\{\int_{0}^{T_{n}} h\left(y_{x}(s), \alpha_{x, n}^{j}\left[b_{n}\right](s), b_{n}(s)\right) d s\right\},
\end{aligned}
$$

and in this case $T_{n} \rightarrow 0$ as $n \rightarrow \infty$. Note that the integral terms tend to 0 uniformly with respect to $b_{n} \in \mathcal{B}$ as $T_{n} \rightarrow 0$ by assumption (A2), the uniform estimate (2.3), and the continuity assumption (A1) on $h$. Thus we have

$$
\liminf _{n \rightarrow \infty}\left\{\sum_{m=1}^{d_{n}} k\left(a_{m-1}, a_{m}\right)\right\} \leq 0
$$

which contradicts (A5).

Lemma 3.5. Assume (A1)-(A6) and $V_{\gamma}^{j} \in C\left(\mathbb{R}^{N}\right), j=1, \ldots, r$. If $V_{\gamma}^{j}(x)<$ $\min _{i \neq j}\left\{V_{\gamma}^{i}(x)+k\left(a^{j}, a^{i}\right)\right\}$, then there exists $\tau=\tau_{x}>0$ such that

$$
V_{\gamma}^{j}(x) \geq \sup _{b \in \mathcal{B}}\left\{\int_{0}^{t}\left[h\left(y_{x}(s), a^{j}, b(s)\right)-\gamma^{2}|b(s)|^{2}\right] d s+V_{\gamma}^{j}\left(y_{x}(t)\right)\right\} \quad \text { for all } t \in\left(0, \tau_{x}\right) .
$$

Proof. From Proposition 3.4, choose $\tau=\tau_{x}>0$ such that, for all $t \in(0, \tau)$,

$$
V_{\gamma}^{j}(x)=\sup _{b \in \mathcal{B}, T>0}\left\{\int_{0}^{t \wedge T}\left[h\left(y_{x}(s), a^{j}, b(s)\right)-\gamma^{2}|b(s)|^{2}\right] d s+1_{[0, T)}(t) V_{\gamma}^{j}\left(y_{x}(t)\right)\right\} .
$$

Thus

$$
\begin{aligned}
V_{\gamma}^{j}(x) & \geq \sup _{b \in \mathcal{B}, T>t}\left\{\int_{0}^{t \wedge T}\left[h\left(y_{x}(s), a^{j}, b(s)\right)-\gamma^{2}|b(s)|^{2}\right] d s+1_{[0, T)}(t) V_{\gamma}^{j}\left(y_{x}(t)\right)\right\} \\
& =\sup _{b \in \mathcal{B}}\left\{\int_{0}^{t}\left[h\left(y_{x}(s), a^{j}, b(s)\right)-\gamma^{2}|b(s)|^{2}\right] d s+V_{\gamma}^{j}\left(y_{x}(t)\right)\right\} .
\end{aligned}
$$

Theorem 3.6. Assume (A1)-(A6) and $V_{\gamma}^{j} \in C\left(\mathbb{R}^{N}\right), j=1, \ldots, r$. Then $V_{\gamma}$ is a viscosity solution of the SQVI (2.5)

$$
\max \left\{H^{j}\left(x, D V_{\gamma}^{j}(x)\right), V_{\gamma}^{j}(x)-\min _{i \neq j}\left\{V_{\gamma}^{i}(x)+k\left(a^{j}, a^{i}\right)\right\}\right\}=0, x \in \mathbb{R}^{N}, j=1, \ldots, r .
$$

Proof. We first show that $V_{\gamma}^{j}$ is a viscosity supersolution of the SQVI (3.18). Fix $x_{0} \in \mathbb{R}^{N}$ and $a^{j} \in A$. Let $\varphi^{j} \in C^{1}\left(\mathbb{R}^{N}\right)$, and $x_{0}$ is a local minimum of $V_{\gamma}^{j}-\varphi^{j}$. We want to show that

$$
\max \left\{H^{j}\left(x_{0}, D \varphi^{j}\left(x_{0}\right)\right), V_{\gamma}^{j}\left(x_{0}\right)-\min _{i \neq j}\left\{V_{\gamma}^{i}\left(x_{0}\right)+k\left(a^{j}, a^{i}\right)\right\}\right\} \geq 0 .
$$

We have two cases to consider.

$$
\text { Case 1. } V_{\gamma}^{j}\left(x_{0}\right)=\min _{i \neq j}\left\{V_{\gamma}^{i}\left(x_{0}\right)+k\left(a^{j}, a^{i}\right)\right\} \text {. }
$$


Case 2. $V_{\gamma}^{j}\left(x_{0}\right)<\min _{i \neq j}\left\{V_{\gamma}^{i}\left(x_{0}\right)+k\left(a^{j}, a^{i}\right)\right\}$.

If Case 1 occurs, we have

$$
\begin{aligned}
& \max \left\{H^{j}\left(x_{0}, D \varphi^{j}\left(x_{0}\right)\right), V_{\gamma}^{j}\left(x_{0}\right)-\min _{i \neq j}\left\{V_{\gamma}^{i}\left(x_{0}\right)+k\left(a^{j}, a^{i}\right)\right\}\right\} \\
& \geq V_{\gamma}^{j}\left(x_{0}\right)-\min _{i \neq j}\left\{V_{\gamma}^{i}\left(x_{0}\right)+k\left(a^{j}, a^{i}\right)\right\} \\
& \geq 0 .
\end{aligned}
$$

If Case 2 occurs, we want to show that $H^{j}\left(x_{0}, D \varphi^{j}\left(x_{0}\right)\right) \geq 0$. Fix $b \in B$, and set $b(s)=b$ for all $s \geq 0$. From Lemma 3.5, choose $\bar{t}_{0}>0$ such that, for $t \in\left(0, \bar{t}_{0}\right)$,

$$
V_{\gamma}^{j}\left(x_{0}\right)-V_{\gamma}^{j}\left(y_{x_{0}}(t)\right) \geq \int_{0}^{t}\left[h\left(y_{x_{0}}(s), a^{j}, b\right)-\gamma^{2}|b|^{2}\right] d s .
$$

Since $x_{0}$ is a local minimum of $V_{\gamma}^{j}-\varphi^{j}$, by (2.3) there exists $\hat{t}_{0}>0$ such that

$$
\varphi^{j}\left(x_{0}\right)-\varphi^{j}\left(y_{x_{0}}(s), a^{j}, b(s)\right) \geq V_{\gamma}^{j}\left(x_{0}\right)-V_{\gamma}^{j}\left(y_{x_{0}}(s), a^{j}, b(s)\right), \quad 0<s<\hat{t}_{0} .
$$

Set $t_{0}=\min \left\{\bar{t}_{0}, \hat{t}_{0}\right\}$. As a consequence of (3.20) and (3.21), we have

$$
\varphi^{j}\left(x_{0}\right)-\varphi^{j}\left(y_{x_{0}}(t)\right) \geq \int_{0}^{t}\left[h\left(y_{x_{0}}(s), a^{j}, b\right)-\gamma^{2}|b|^{2}\right] d s, \quad 0<t<t_{0} .
$$

Divide both sides by $t$, and let $t \rightarrow 0$ to get

$$
-D \varphi^{j}\left(x_{0}\right) \cdot f\left(x_{0}, a^{j}, b\right)-h\left(x_{0}, a^{j}, b\right)+\gamma^{2}|b|^{2} \geq 0 .
$$

Since $b \in B$ is arbitrary, we have $H^{j}\left(x_{0}, D \varphi^{j}\left(x_{0}\right)\right) \geq 0$.

We next show that $V_{\gamma}^{j}$ is a viscosity subsolution of the SQVI (3.18). Fix $x_{1} \in \mathbb{R}^{N}$ and $a^{j} \in A$. Let $\varphi^{j} \in C^{1}\left(\mathbb{R}^{N}\right)$, and $x_{1}$ is a local maximum of $V_{\gamma}^{j}-\varphi^{j}$. We want to show that

$$
\max \left\{H^{j}\left(x_{1}, D \varphi^{j}\left(x_{1}\right)\right), V_{\gamma}^{j}\left(x_{1}\right)-\min _{i \neq j}\left\{V_{\gamma}^{i}\left(x_{1}\right)+k\left(a^{j}, a^{i}\right)\right\}\right\} \leq 0 .
$$

From Proposition 3.1, $V_{\gamma}^{j}\left(x_{1}\right) \leq \min _{i \neq j}\left\{V_{\gamma}^{i}\left(x_{1}\right)+k\left(a^{j}, a^{i}\right)\right\}$. Thus we want to show that $H^{j}\left(x_{1}, D \varphi^{j}\left(x_{1}\right)\right) \leq 0$.

Let $t>0$ and $\epsilon>0$. From (3.12) in Corollary 3.3, we may choose $\hat{b}=\hat{b}_{t, \epsilon} \in \mathcal{B}$ such that

$$
V_{\gamma}^{j}\left(x_{1}\right) \leq \int_{0}^{t}\left[h\left(y_{x_{1}}(s), a^{j}, \hat{b}(s)\right)-\gamma^{2}|\hat{b}(s)|^{2}\right] d s+V_{\gamma}^{j}\left(y_{x_{1}}(t, \hat{b})\right)+\epsilon t,
$$

and hence

$$
V_{\gamma}^{j}\left(x_{1}\right)-V_{\gamma}^{j}\left(y_{x_{1}}(t, \hat{b})\right) \leq \int_{0}^{t}\left[h\left(y_{x_{1}}(s), a^{j}, \hat{b}(s)\right)-\gamma^{2}|\hat{b}(s)|^{2}\right] d s+\epsilon t .
$$

Since $x_{1}$ is a local maximum of $V_{\gamma}^{j}-\varphi^{j}$, by (2.3) we may assume that

$$
\varphi^{j}\left(x_{1}\right)-\varphi^{j}\left(y_{x_{1}}(s), a^{j}, \hat{b}(s)\right) \leq V_{\gamma}^{j}\left(x_{1}\right)-V_{\gamma}^{j}\left(y_{x_{1}}(s), a^{j}, \hat{b}(s)\right), \quad 0<s \leq t .
$$


Combine (3.25) and (3.26) to get

$$
\varphi^{j}\left(x_{1}\right)-\varphi^{j}\left(y_{x_{1}}\left(t, a^{j}, \hat{b}(t)\right)\right) \leq \int_{0}^{t}\left[h\left(y_{x_{1}}(s), a^{j}, \hat{b}(s)\right)-\gamma^{2}|\hat{b}(s)|^{2}\right] d s+\epsilon t .
$$

Observe that (2.3) and (A3) imply

$$
\left|f\left(y_{x_{1}}(s), a^{j}, \hat{b}(s)\right)-f\left(x_{1}, a^{j}, \hat{b}(s)\right)\right| \leq \omega_{f}\left(M_{x} s,|x|+M_{x} t_{0}\right) \text { for } 0<s<t_{0}
$$

and

$$
\left|h\left(y_{x}(s), a^{j}, \hat{b}(s)\right)-h\left(x_{1}, a^{j}, \hat{b}(s)\right)\right| \leq \omega_{h}\left(M_{x_{1}} s,|x|+M_{x_{1}} t_{0}\right) \text { for } 0<s<t_{0},
$$

where $t_{0}$ does not depend on $\epsilon, t$, or $\hat{b}$. By (3.29), the integral on the right-hand side of (3.27) can be written as

$$
\int_{0}^{t}\left[h\left(x_{1}, a^{j}, \hat{b}(s)\right)-\gamma^{2}|\hat{b}(s)|^{2}\right] d s+\mathrm{o}(t) \text { as } t \rightarrow 0 .
$$

Thus

$$
\varphi^{j}\left(x_{1}\right)-\varphi^{j}\left(y_{x_{1}}\left(t, a^{j}, \hat{b}(t)\right)\right) \leq \int_{0}^{t}\left[h\left(x_{1}, a^{j}, \hat{b}(s)\right)-\gamma^{2}|\hat{b}(s)|^{2}\right] d s+\epsilon t+\mathrm{o}(t) .
$$

Moreover,

$$
\begin{aligned}
\varphi^{j}\left(x_{1}\right)-\varphi^{j}\left(y_{x_{1}}\left(t, a^{j}, \hat{b}\right)\right) & =-\int_{0}^{t} \frac{d}{d s} \varphi^{j}\left(y_{x_{1}}\left(s, a^{j}, \hat{b}\right)\right) d s \\
& =-\int_{0}^{t} D \varphi^{j}\left(y_{x_{1}}\left(s, a^{j}, \hat{b}\right)\right) \cdot f\left(y_{x_{1}}(s), a^{j}, \hat{b}(s)\right) d s \\
& =-\int_{0}^{t} D \varphi^{j} \cdot f\left(x_{1}, a^{j}, \hat{b}(s)\right) d s+\mathrm{o}(t)
\end{aligned}
$$

where we used (2.3), (3.28), and $\varphi^{j} \in C^{1}$ in the last equality to estimate the difference between $D \varphi^{j} \cdot f$ computed at $y_{x_{1}}(s)$ and at $x_{1}$, respectively. Plugging (3.31) into (3.30) gives

$$
\int_{0}^{t}-D \varphi^{j}\left(x_{1}\right) \cdot f\left(x_{1}, a^{j}, \hat{b}(s)\right) d s \leq \int_{0}^{t}\left[h\left(x_{1}, a^{j}, \hat{b}(s)\right)-\gamma^{2}|\hat{b}|^{2}\right] d s+\epsilon t+\mathrm{o}(t) .
$$

Thus

$$
\int_{0}^{t}\left[-D \varphi^{j}\left(x_{1}\right) \cdot f\left(x_{1}, a^{j}, \hat{b}(s)\right)-h\left(x_{1}, a^{j}, \hat{b}(s)\right)+\gamma^{2}|\hat{b}(s)|^{2}\right] d s \leq \epsilon t+\mathrm{o}(t) .
$$

We estimate the left-hand side of this inequality from below next to get

$$
\inf _{b \in B}\left\{-D \varphi^{j}\left(x_{1}\right) \cdot f\left(x_{1}, a^{j}, b\right)-h\left(x_{1}, a^{j}, b\right)+\gamma^{2}|b|^{2}\right\} \cdot t \leq \epsilon t+o(t) .
$$

Divide by $t$, and pass to the limit as $t \rightarrow 0$ to get

$$
\inf _{b \in B}\left\{-D \varphi^{j}(x) \cdot f\left(x, a^{j}, b\right)-h\left(x, a^{j}, b\right)+\gamma^{2}|b|^{2}\right\} \leq \epsilon .
$$


Since $\epsilon>0$ is arbitrary, we conclude that $H^{j}\left(x, D \varphi^{j}(x)\right) \leq 0$.

We next give a connection of a switching storage (vector) function with the SQVI (3.18).

TheOrem 3.7. Assume (A1)-(A5), and assume that $S=\left(S^{1}, \ldots, S^{r}\right)$ is a continuous switching-storage function for the closed-loop system formed by the nonanticipating strategy $\alpha \in \Gamma$. Then $S$ is a viscosity supersolution of SQVI (3.18).

Proof. The proof follows exactly as the proof that the lower-value function $V^{\gamma}$ is a viscosity subsolution in the proof of Theorem 3.6 once we verify the following analogue of Lemma 3.5 for switching-storage functions.

Lemma 3.8. Assume (A1)-(A6), and assume that $S=\left(S^{1}, \ldots, S^{r}\right)$ is a continuous switching-storage function. If $S^{j}(x)<\min _{i \neq j}\left\{S^{i}(x)+k\left(a^{j}, a^{i}\right)\right\}$, then there exists $\tau=\tau_{x}>0$ such that

$$
S^{j}(x) \geq \sup _{b \in \mathcal{B}}\left\{\int_{0}^{t}\left[h\left(y_{x}(s), a^{j}, b(s)\right)-\gamma^{2}|b(s)|^{2}\right] d s+S^{j}\left(y_{x}(t)\right)\right\} \quad \text { for all } t \in\left(0, \tau_{x}\right) .
$$

Proof. By the defining condition (1.5) for a storage function (1.5) (with attenuation level $\gamma$ ), we have

$$
\begin{aligned}
S^{j}(x) \geq \int_{0}^{t}\left[h\left(y_{x}(s), \alpha_{x}^{j}[b](s), b(s)\right)-\gamma^{2}|b(s)|^{2}\right] d s \\
+\sum_{0 \leq \tau<t} k\left(\alpha_{x}^{j}[b]\left(\tau^{-}\right), \alpha_{x}^{j}[b](\tau)\right)+S^{j(t)}\left(y_{x}\left(t, \alpha_{x}^{j}[b], b\right)\right) .
\end{aligned}
$$

Due to the assumed boundedness of $B$ and the boundedness of $h$ (see (A2)), it is clear that, given $\epsilon>0$, we may choose $\tau=\tau_{\epsilon}$ so that

$$
\sup _{b \in \mathcal{B}}\left\{\int_{0}^{t}\left[h\left(y_{x}(s), a^{j}, b(s)\right)-\gamma^{2}|b(s)|^{2}\right] d s\right\}>-\epsilon
$$

for all $t \in\left[0, \tau_{\epsilon}\right)$. We conclude that, for any such $t$,

$$
S^{j}(x) \geq-\epsilon+\sum_{0 \leq \tau<t} k\left(\alpha_{x}^{j}[b]\left(\tau^{-}\right), \alpha_{x}^{j}[b](\tau)\right)+S^{j(t)}\left(y_{x}\left(t, \alpha_{x}^{j}[b], b\right)\right) .
$$

If we now choose $\epsilon=\frac{1}{2}\left[\min _{i \neq j}\left\{S^{i}(x)+k\left(a^{j}, a^{i}\right)\right\}-S^{j}(x)\right]>0$ and use the continuity of $S^{j}$ for each $j$, the estimate (3.35) in the presence of any jumps in the interval $\left[0, \tau_{\epsilon}\right)$ leads to a contradiction. Since we are now assured that there are no jumps, (3.34) collapses to

$$
S^{j}(x) \geq \int_{0}^{t}\left[h\left(y_{x}(s), \alpha_{x}^{j}[b](s), b(s)\right)-\gamma^{2}|b(s)|^{2}\right] d s+S^{j}\left(y_{x}\left(t, \alpha_{x}^{j}[b], b\right)\right)
$$

for $0 \leq t<\tau_{\epsilon}$ and for all $b \in \mathcal{B}$. Taking the supremum of $b \in \mathcal{B}$ now leads to the desired result. This concludes the proof of Lemma 3.8 and of Theorem 3.7.

We now proceed to the synthesis of a switching-control strategy achieving the dissipation inequality for a given viscosity supersolution $U=\left(U^{1}, \ldots, U^{r}\right)$ of SQVI (3.18). Given a continuous nonnegative vector function $U=\left(U^{1}, \ldots, U^{r}\right)$ on $\mathbb{R}^{N}$ satisfying the condition

$$
U^{j}(x) \leq \min _{i \neq j}\left\{U^{i}(x)+k\left(a^{j}, a^{i}\right)\right\} \text { for all } x \in \mathbb{R}^{N}, j=1, \ldots, r,
$$


we associate a state-feedback switching strategy $\alpha_{U}:\left(y(t), a^{j}\right) \rightarrow \alpha^{j}(y(t))$ by the rule

$$
\alpha^{j}(y(t))= \begin{cases}a^{j} & \text { if } U^{j}(y(t))<\min _{i \neq j}\left\{U^{i}(y(t))+k\left(a^{j}, a^{i}\right)\right\}, \\ a^{\ell} & \text { for any } \ell \in \arg \min _{i \neq j}\left\{U^{i}(y(t))+k\left(a^{j}, a^{i}\right)\right\} \text { otherwise. }\end{cases}
$$

In other words, the associated feedback switching strategy is as follows: if the current state is $y(t)$ and the current old control is $a\left(t^{-}\right)=a^{j}$, then set $a(t)=\alpha^{j}(y(t))$. Such a strategy can also be expressed as a nonanticipating strategy $\alpha_{U}:\left(x, a^{j}, b\right) \rightarrow \alpha_{U, x}^{j}[b]$; explicitly, for this particular case $\alpha_{U}$, we have that $\alpha_{U, x}^{j}[b]$ is given by

$$
\alpha_{U, x}^{j}[b](t)=\sum_{n \geq 1} a_{n-1} 1_{\left[\tau_{n-1}, \tau_{n}\right)}(t) \text { for } t \geq 0
$$

and $\alpha_{U, x}^{j}[b]\left(0^{-}\right)=a_{0}$, where

$$
\tau_{0}=0, a_{0}=a^{j_{0}}=a^{j},
$$

and, for $n=1,2,3, \ldots, \tau_{n}[b]$ is the infimum over $t>\tau_{n-1}$ for which

$$
\begin{aligned}
U^{j_{n-1}}\left(y_{y\left(\tau_{n-1}\right)}\right. & \left.\left(t-\tau_{n-1}, a^{j_{n-1}}, b\left(\cdot-\tau_{n-1}\right)\right)\right) \\
= & \left.\min _{i \neq j_{n-1}}\left\{U^{i}\left(y_{y\left(\tau_{n-1}\right)}\left(t-\tau_{n-1}, a^{j_{n-1}}, b\left(\cdot-\tau_{n-1}\right)\right)\right)+k\left(a^{j_{n-1}}, a^{i}\right)\right\}\right\},
\end{aligned}
$$

or $+\infty$ if the preceding set is empty; and $a_{n}=a^{j_{n}}=$ any $a^{l} \neq a^{j_{n-1}}$ for which

$$
\begin{aligned}
\min _{i \neq j_{n-1}}\left\{U ^ { i } \left(y _ { y ( \tau _ { n - 1 } ) } \left(\tau_{n}\right.\right.\right. & \left.\left.\left.-\tau_{n-1}, a^{j_{n-1}}, b\left(\cdot-\tau_{n-1}\right)\right)\right)+k\left(a^{j_{n-1}}, a^{i}\right)\right\} \\
& =U^{l}\left(y_{y\left(\tau_{n-1}\right)}\left(\tau_{n}-\tau_{n-1}, a^{j_{n-1}}, b\left(\cdot-\tau_{n-1}\right)\right)\right)+k\left(a^{j_{n-1}}, a^{l}\right)
\end{aligned}
$$

if $\tau_{n}<\infty$ or undefined if $\tau_{n}=\infty$. Note that, if $\tau_{1}=\tau_{0}=0$, there is an immediate switch from $a_{0}$ to $a_{1}$ at time 0 , and the $n=1$ term in (3.37) is vacuous. Moreover, by (A5), $\tau_{n}>\tau_{n-1}$ for $\tau_{n-1}<\infty$, and $n>1$. To see this, we assume that $\tau_{n}=\tau_{n-1}<\infty$ for some $n>1$. From the definition of $\tau_{n-1}$ and $\tau_{n}$, we would have

$$
\begin{aligned}
U^{j_{n-2}}\left(y\left(\tau_{n-1}\right)\right) & =U^{j_{n-1}}\left(y\left(\tau_{n-1}\right)\right)+k\left(a^{j_{n-2}}, a^{j_{n-1}}\right) \\
& \left.=U^{j_{n}}\left(y\left(\tau_{n-1}\right)\right)+k\left(a^{j_{n-1}}, a^{j_{n}}\right)+k\left(a^{j_{n-2}}, a^{j_{n-1}}\right) \text { (hence } j_{n} \neq j_{n-2}\right) \\
& >U^{j_{n}}\left(y\left(\tau_{n-1}\right)\right)+k\left(a^{j_{n-2}}, a^{j_{n}}\right) \\
& \geq \min _{i \neq j_{n-2}}\left\{U^{i}\left(y\left(\tau_{n-1}\right)\right)+k\left(a^{j_{n-2}}, a^{i}\right)\right\},
\end{aligned}
$$

which gives a contradiction. Moreover, as shown in the proof of the next theorem, if $\tau_{n}<\infty$ for all $n$, it still holds that $\lim _{n \rightarrow \infty} \tau_{n}=\infty$, so the closed-loop trajectory is defined for all $t>0$.

TheOREM 3.9. Assume the following.

(i) (A1)-(A5) hold.

(ii) $U=\left(U^{1}, \ldots, U^{r}\right)$ is a nonnegative continuous viscosity supersolution in $\mathbb{R}^{N}$ of the $S Q V I(3.18)$

$$
\max \left\{H^{j}\left(x, D U^{j}(x)\right), U^{j}(x)-\min _{i \neq j}\left\{U^{i}(x)+k\left(a^{j}, a^{i}\right)\right\}\right\}=0, x \in \mathbb{R}^{N}, j=1, \ldots, r .
$$


(iii) $U^{j}(x) \leq \min _{i \neq j}\left\{U^{i}(x)+k\left(a^{j}, a^{i}\right)\right\}, x \in \mathbb{R}^{N}, j \in\{1, \ldots, r\}$.

Let $\alpha_{U}$ be the state-feedback strategy defined by (3.36) or, equivalently, the nonanticipating disturbance-feedback strategy $\alpha_{U}$ defined by (3.37). Then $U=\left(U^{1}, \ldots, U^{r}\right)$ is a storage function for the closed-loop system formed by the strategy $\alpha_{U}$. In particular, we have

$$
U^{j}(x) \geq \sup _{b \in \mathcal{B}, T \geq 0}\left\{\int_{[0, T)} l\left(y_{x}(s), a^{j}, \alpha_{U, x}^{j}[b](s), b(s)\right)\right\} \geq V_{\gamma}^{j}(x)
$$

for each $x \in \mathbb{R}^{N}$ and $a^{j} \in A$. Thus $V_{\gamma}$, if continuous, is characterized as the minimal, nonnegative continuous viscosity supersolution of the SQVI (3.18) satisfying condition (iii) as well as the minimal continuous switching-storage function satisfying condition (iii) for the closed-loop system associated with some nonanticipating strategy $\alpha_{V_{\gamma}}$.

Proof. Let $\alpha_{U, x}^{j}[b](t)$ be the switching strategy defined as in (3.37). We claim that

$$
\tau_{n} \rightarrow \infty \text { as } n \rightarrow \infty \text {. }
$$

If $\tau_{n}=\infty$ for some $n$, then it is trivially true. Otherwise, since we observed just before the statement of Theorem 3.9 that $\left\{\tau_{n}\right\}$ is a nondecreasing sequence, it would follow that

$$
\lim _{n \rightarrow \infty} \tau_{n}=T<\infty
$$

with $0 \leq \tau_{n}<T$ for all $n$. From (3.38), we have that $\left\{\tau_{n}\right\}$ is a Cauchy sequence, and hence for all $\nu>0$ there is some $n$ such that $\tau_{n}<\tau_{n-1}+\nu$. By the definition of $\tau_{n}$,

$$
U^{j_{n-1}}\left(y_{x}\left(\tau_{n}\right)\right)=U^{l}\left(y_{x}\left(\tau_{n}\right)\right)+k\left(a^{j_{n-1}}, a^{l}\right) \text { for some } a^{l} \neq a^{j_{n-1}} .
$$

(We have written $y_{x}(t)$ for $y_{x}\left(t, \alpha_{x}^{j}[b], b\right)$.) By definition of $\tau_{n-1}$, we have

$$
U^{j_{n-2}}\left(y_{x}\left(\tau_{n-1}\right)\right)=U^{j_{n-1}}\left(y_{x}\left(\tau_{n-1}\right)\right)+k\left(a^{j_{n-2}}, a^{j_{n-1}}\right) .
$$

By (iii), we have

$$
\begin{aligned}
U^{j_{n-2}}\left(y_{x}\left(\tau_{n-1}\right)\right) & \leq \min _{i \neq j_{n-2}}\left\{U^{i}\left(y_{x}\left(\tau_{n-1}\right)\right)+k\left(a^{j_{n-2}}, a^{i}\right)\right\} \\
& \leq U^{l}\left(y_{x}\left(\tau_{n-1}\right)\right)+k\left(a^{j_{n-2}}, a^{l}\right) \text { if } l \neq j_{n-2},
\end{aligned}
$$

and hence

$$
U^{j_{n-2}}\left(y_{x}\left(\tau_{n-1}\right)\right) \leq U^{l}\left(y_{x}\left(\tau_{n-1}\right)\right)+k\left(a^{j_{n-2}}, a^{l}\right)
$$

if $l \neq j_{n-2}$. If $l=j_{n-2},(3.41)$ holds with equality (by (A5)), and hence (3.41) in fact holds without restriction. From (3.40) and (3.41), we have

$$
k\left(a^{j_{n-2}}, a^{j_{n-1}}\right)-k\left(a^{j_{n-2}}, a^{l}\right) \leq U^{l}\left(y_{x}\left(\tau_{n-1}\right)\right)-U^{j_{n-1}}\left(y_{x}\left(\tau_{n-1}\right)\right) .
$$

As a consequence of (3.39) and (3.42), we have

$$
\begin{aligned}
0 & <k\left(a^{j_{n-2}}, a^{j_{n-1}}\right)+k\left(a^{j_{n-1}}, a^{l}\right)-k\left(a^{j_{n-2}}, a^{l}\right) \\
& \leq U^{l}\left(y_{x}\left(\tau_{n-1}\right)\right)-U^{l}\left(y_{x}\left(\tau_{n}\right)\right)+U^{j_{n-1}}\left(y_{x}\left(\tau_{n}\right)\right)-U^{j_{n-1}}\left(y_{x}\left(\tau_{n-1}\right)\right) \\
& \leq \omega_{l}(\nu)+\omega_{j_{n-1}}(\nu),
\end{aligned}
$$


and hence (by the strict triangle inequality in (A5))

$$
0<\min _{i, j, l: i \neq j \neq l}\left\{k\left(a^{i}, a^{j}\right)+k\left(a^{j}, a^{l}\right)-k\left(a^{i}, a^{l}\right)\right\} \leq \omega_{\ell}(\nu)+\omega_{j}(\nu),
$$

where, in general, $\omega_{j}$ is a modulus of continuity for $U^{j}\left(y_{x}(\cdot)\right)$ on the interval $[0, T]$. Letting $\nu$ tend to zero now leads to a contradiction, and the claim follows.

Hence $\alpha_{x}^{j}[b](t)=\sum a_{n-1} 1_{\left[\tau_{n-1}, \tau_{n}\right)}(t) \in \Gamma$. Since $U$ is a viscosity supersolution of the SQVI (3.18), we have $H^{j_{n}}\left(y_{x}(s), D U^{j_{n}}\left(y_{x}(s)\right)\right) \geq 0$ in the viscosity-solution sense for $\tau_{n}<s<\tau_{n+1}$. Thus (see [6, section II.5.5])

$$
U^{j_{n}}\left(y_{x}(s)\right)-U^{j_{n}}\left(y_{x}(t)\right) \geq \int_{s}^{t}\left[h\left(y_{x}(s), a^{j_{n}}, b(s)\right)-\gamma^{2}|b(s)|^{2}\right] d s
$$

for all $b \in \mathcal{B}, \tau_{n}<s \leq t<\tau_{n+1}$. (This argument uses the boundedness of the disturbance set $B$.) Letting $s \rightarrow \tau_{n}^{+}$and $t \rightarrow \tau_{n+1}^{-}$, we get

$$
U^{j_{n}}\left(y_{x}\left(\tau_{n}\right)\right)-U^{j_{n}}\left(y_{x}\left(\tau_{n+1}\right)\right) \geq \int_{\tau_{n}}^{\tau_{n+1}}\left[h\left(y_{x}(s), a^{j_{n}}, b(s)\right)-\gamma^{2}|b(s)|^{2}\right] d s \quad \text { for all } b \in \mathcal{B} \text {. }
$$

We also have

$$
U^{j_{n}}\left(y_{x}\left(\tau_{n+1}\right)\right)=U^{j_{n+1}}\left(y_{x}\left(\tau_{n+1}\right)\right)+k\left(a^{j_{n}}, a^{j_{n+1}}\right) \text { for } \tau_{n+1}<\infty .
$$

Adding (3.44) over $\tau_{n} \leq T$ and using (3.45), we have

$$
\begin{aligned}
U^{j_{0}}(x) & \geq \int_{0}^{T}\left[h\left(y_{x}(s), \alpha_{x}^{j}[b](s), b(s)\right)-\gamma^{2}|b(s)|^{2}\right] d s+\sum_{\tau_{n} \leq T} k\left(a_{n-1}, a_{n}\right)+U^{j_{n}}\left(y_{x}(T)\right) \\
& \geq \int_{0}^{T}\left[h\left(y_{x}(s), \alpha_{x}^{j}[b](s), b(s)\right)-\gamma^{2}|b(s)|^{2}\right] d s+\sum_{\tau_{n} \leq T} k\left(a_{n-1}, a_{n}\right) .
\end{aligned}
$$

Since this inequality holds for arbitrary $b \in \mathcal{B}$ and $T \geq 0$, we have

$$
U^{j}(x) \geq \sup _{b \in \mathcal{B}, T \geq 0}\left\{\int_{[0, T]} l\left(y_{x}(s), a^{j}, \alpha_{x}^{j}[b](s), b(s)\right)\right\} .
$$

Thus $U^{j}(x) \geq V_{\gamma}^{j}(x)$. By Theorem 3.6, we know that $V_{\gamma}$ is a viscosity supersolution of the SQVI (3.18) if it is continuous. (Note that the proof of the viscosity-supersolution property of $V_{\gamma}$ in Theorem 3.6 does not use the assumption (A6).) Also, $V_{\gamma}$ has the property (iii) by Proposition 3.1. Thus we conclude that, if continuous, $V_{\gamma}$ is the minimal, nonnegative continuous viscosity supersolution of SQVI (3.18) which satisfies condition (iii)

The first part of Theorem 3.9, already proved, then implies that $V_{\gamma}$ is a switchingstorage function. Moreover, if $S$ is any continuous, switching-storage function for some nonanticipating strategy $\alpha_{V_{\gamma}}$, from Theorem 3.7 we see that $S$ is a viscosity supersolution of the SQVI (3.18). Again, from the first part of this theorem, already proved, we then see that $S \geq V_{\gamma}$ if $S$ has the property (iii), and hence $V_{\gamma}$ is also the minimal continuous switching-storage function satisfying the condition (iii), as asserted. 
Remark 1. The proof of Theorem 3.9 required deduction of an integral inequality (3.43) from knowledge of an inequality of the form $H(y(x), D U(y(x)) \geq 0$ holding in the viscosity sense; the proof of this fact from [6, section II.5.5] ultimately uses the boundedness of the disturbance set $B$. However, the paper [13] obtains such an integral inequality without a boundedness assumption by using other tools of nonsmooth analysis (e.g., "contingent epiderivative" and viability theory). By using this alternative nonsmooth framework rather than restricting oneself to "viscositysense supersolutions," one can get a version of Theorem 3.9 which does not rely on the boundedness of $B$.

Remark 2. The results of this section reduce the computation of robust statefeedback switching strategy $\alpha$ to computing the solution $\left(U=U^{1}, \ldots, U^{r}\right)$ (or, more precisely, the minimal viscosity supersolution) of the SQVI of the form

(SQVI)

$$
\max \left\{H^{j}\left(x, D U^{j}(x)\right), U^{j}(x)-\min _{i \neq j}\left\{U^{i}(x)+k\left(a^{j}, a^{i}\right)\right\}\right\}=0, \quad j=1, \ldots, r .
$$

This leaves open the issue of how one computes such a solution of an SQVI. A connection can be made with the easier problem of solving a single variational inequality as follows.

If $U=\left(U^{1}, \ldots, U^{r}\right)$ (with $U^{j} \in C\left(\mathbb{R}^{N}\right)$ for $j=1, \ldots, r$ ) is the minimal viscosity supersolution of (SQVI), then each $U^{j}$ can be interpreted as the minimal viscosity supersolution of the variational inequality (VI)

$$
\max \{H(x, D U(x)), U(x)-\Phi(x)\}=0
$$

with Hamiltonian $H$ equal to $H^{j}$ and with stopping cost $\Phi$ equal to $\Phi^{j}=\min _{i \neq j}\left\{U^{i}+\right.$ $\left.k\left(a^{j}, a^{i}\right)\right\}$. This suggests defining an iteration map $F$ as follows. Given an $r$-tuple $U=\left(U^{1}, \ldots, U^{r}\right)$ of nonnegative real-valued functions, define a new $r$-tuple $F(U)=$ $\left(F(U)^{1}, \ldots, F(U)^{r}\right)$ of nonnegative real-valued functions by

$$
F(U)^{j}=\text { the minimal viscosity supersolution of (VI) with } H=H^{j} \text { and } \Phi=\Phi^{j} .
$$

Note that $U$ is the minimal viscosity supersolution of (SQVI) if and only if $F(U)=U$, i.e., if and only if $U$ is a fixed point of $F$. Formally, one can solve the fixed point problem by guessing a starting point $U_{0}=\left(U_{0}^{1}, \ldots, U_{0}^{r}\right)$ and then iterating

$$
U_{n+1}=F\left(U_{n}\right), n=0,1,2, \ldots, .
$$

If $U_{n} \rightarrow U_{\infty}$ and $F$ is continuous, then, from $U_{n+1}=F\left(U_{n}\right)$, one can take the limit to get $U_{\infty}=F\left(U_{\infty}\right)$, from which we see that $U_{\infty}$ is a fixed point for $F$. For finite horizon problems or problems with a positive discount factor in the running cost, the connection is a little cleaner; in this situation, one has a uniqueness theorem for solutions of the relevant SQVI.

A similar remark giving a connection between the impulsive control problem and the stopping time problem is given in [6, Chapter III, section 4.3], where some convergence results are also given. It would be of interest to develop similar convergence results for the SQVI associated with an optimal switching-control problem.

4. Stability for switching-control problems. In this section, we show how the solution of the SQVI (3.18) can be used for stability analysis. 
We consider the system (1.1)-(1.2) with some control strategy $\alpha$ plugged in to get a closed-loop system with the disturbance signal as the only input:

$$
\Sigma_{s w}\left\{\begin{aligned}
\dot{y} & =f\left(y, \alpha_{x}^{j}[b], b\right), y(0)=x, a\left(0^{-}\right)=a^{j}, \\
z & =h\left(y, \alpha_{x}^{j}[b], b\right) .
\end{aligned}\right.
$$

An example of such a strategy $\alpha$ is the canonical strategy $\alpha_{U}$ (see (3.36) or (3.37)) determined by a continuous supersolution of the SQVI (3.18). Moreover, if $V_{\gamma}=$ $\left(V_{\gamma}^{1}, \ldots, V_{\gamma}^{r}\right)$ is the vector lower-value function for the associated game as in (1.4) and we assume that 0 is an equilibrium point for the autonomous system formed from $(1.1)-(1.2)$ by taking $a(s)=a^{i_{0}}$ and $b(s)=0\left(\right.$ so $f\left(0, a^{i_{0}}, 0\right)=0$ and $\left.h\left(0, a^{i_{0}}, 0\right)=0\right)$, then it is easy to check that $V_{\gamma}^{i_{0}}(0)=0$. Furthermore, the associated strategy $\alpha=\alpha_{V_{\gamma}}$ has the property that

$$
\alpha_{0}^{i_{0}}[0]=a^{i_{0}},
$$

so 0 is an equilibrium point of the closed-loop system $\Sigma_{s w}$ with $\alpha=\alpha_{V_{\gamma}}$ and $a\left(0^{-}\right)=$ $a^{i_{0}}$ as well. Our goal is to give conditions which guarantee a sort of converse, starting with any continuous supersolution $U$ of the SQVI (3.18).

We first need a few preliminaries. The following elementary result can be found, e.g., in [19].

LEMma 4.1. If $\phi(\cdot): \mathbb{R} \rightarrow \mathbb{R}$ is a nonnegative, uniformly continuous function such that $\int_{0}^{\infty} \phi(s) d s<\infty$, then $\lim _{t \rightarrow \infty} \phi(t)=0$.

We say that the closed-loop switching system $\Sigma_{s w}$ is zero-state observable for initial control setting $a^{j}$ if whenever $h\left(y_{x}(t), \alpha_{x}^{j}[0](t), 0\right)=0$ for all $t \geq 0$, then $y_{x}(t)=$ $y_{x}\left(t, \alpha_{x}^{j}[0], 0\right)=0$ for all $t \geq 0$. We say that the closed-loop system $\Sigma_{s w}$ is zero-state detectable for initial control setting $a^{j}$ if

$$
\lim _{t \rightarrow \infty} h\left(y_{x}(t), \alpha_{x}^{j}[0](t), 0\right)=0 \text { implies that } \lim _{t \rightarrow \infty} y_{x}\left(t, \alpha_{x}^{j}[0], 0\right)=0 .
$$

The following proposition gives conditions which guarantee that a particular component $U^{j}$ of a viscosity supersolution $U=\left(U^{1}, \ldots, U^{r}\right)$ is positive-definite, a conclusion which will be needed as a hypothesis in the stability theorem to follow.

Proposition 4.2. Assume the following:

(i) (A1)-(A6) hold;

(ii) $\Sigma_{s w}$ is zero-state observable for some initial control setting $a^{j}$;

(iii) $U=\left(U^{1}, \ldots, U^{r}\right)$ is a nonnegative continuous viscosity supersolution of the $S Q V I(3.18)$

$\max \left\{H^{j}\left(x, D U^{j}(x)\right), U^{j}(x)-\min _{i \neq j}\left\{U^{i}(x)+k\left(a^{j}, a^{i}\right)\right\}\right\}=0, \quad x \in \mathbb{R}^{N}, j=1, \ldots, r ;$

(iv) $U^{j}(x) \leq \min _{i \neq j}\left\{U^{i}(x)+k\left(a^{j}, a^{i}\right)\right\}, x \in \mathbb{R}^{N}, j=1, \ldots, r$.

Then $U^{j}(x)>0$ for $x \neq 0$.

Proof. Let $x \in \mathbb{R}^{N}$. By Theorem 3.9, $U$ is a storage function for $\Sigma_{s w}$ if we use $\alpha=\alpha_{U}$ given by (3.36) or, equivalently, (3.37). Thus

$$
\begin{aligned}
U^{j}(x) & \geq \int_{[0, T)} l\left(y_{x}(s), a^{j}, \alpha_{U, x}^{j}[0](s), 0\right) d s+U^{j(T)}\left(y_{x}\left(T, \alpha_{U, x}^{j}[0], 0\right)\right) \\
& \geq \int_{[0, T)} l\left(y_{x}(s), a^{j}, \alpha_{U, x}^{j}[0](s), 0\right) d s \text { for all } T>0 .
\end{aligned}
$$


Since $k$ is nonnegative, we have

$$
U^{j}(x) \geq \int_{0}^{T} h\left(y_{x}(s), \alpha_{x}^{j}[0](s), 0\right) d s \text { for all } T \geq 0 .
$$

Thus, if $U^{j}(x)=0$, then $h\left(y_{x}\left(s, \alpha_{x}^{j}[0], 0\right), \alpha_{x}^{j}[0](s), 0\right)=0$ for all $s \geq 0$ because $h$ is nonnegative by assumption (A6). Since $\Sigma_{s w}$ is zero-state observable for initial control setting $a^{j}$, it follows that $y_{x}\left(s, \alpha_{x}^{j}[0], 0\right)=0$ for all $s \geq 0$. Thus $x=y_{x}\left(0, \alpha_{x}[0], 0\right)=0$. Since $U^{j}$ is nonnegative, we conclude that, if $x \neq 0$, then $U^{j}(x)>0$.

Proposition 4.3. Assume the following:

(i) (A1)-(A6) hold;

(ii) $U=\left(U^{1}, \ldots, U^{r}\right)$ is a nonnegative continuous viscosity supersolution of the $S Q V I(3.18)$

$$
\max \left\{H^{j}\left(x, D U^{j}(x)\right), U^{j}(x)-\min _{i \neq j}\left\{U^{i}(x)+k\left(a^{j}, a^{i}\right)\right\}\right\}=0, \quad x \in \mathbb{R}^{N}, j=1, \ldots, r ;
$$

(iii) $U^{j}(x) \leq \min _{i \neq j}\left\{U^{i}(x)+k\left(a^{j}, a^{i}\right)\right\}, x \in \mathbb{R}^{N}, j=1, \ldots, r$;

(iv) there is an $i_{0} \in\{1, \ldots, r\}$ such that $U^{i_{0}}(0)=0$ and $U^{i_{0}}(x)>0$ for $x \neq 0$.

(v) $\Sigma_{s w}$ is zero-state detectable for all initial control settings $a^{j} \in A$.

Then the strategy $\alpha_{U}$ associated with $U$ as in (3.36) or (3.37) is such that $\alpha_{U, 0}^{i_{0}}[0](s)=$ $a^{i_{0}}$ for all $s$ and 0 is an equilibrium point for the system $\dot{y}=f\left(y, a_{0}^{i}, 0\right)$. Moreover, 0 is a globally asymptotically stable equilibrium point for the system $\Sigma_{s w}$ in the sense that the solution $y(t)=y_{x}^{j}\left(t, \alpha_{U, x}^{j}[0], 0\right)$ of

$$
\dot{y}=f\left(y, \alpha_{U, x}^{j}[0], 0\right), \quad y(0)=x,
$$

has the property that

$$
\lim _{t \rightarrow \infty} y_{x}^{j}\left(t, \alpha_{U, x}^{j}[0], 0\right)=0
$$

for all $x \in \mathbb{R}^{N}$ and all $a^{j} \in A$.

Proof. Suppose that $U^{i_{0}}(0)=0$ and $U^{i_{0}}(x)>0$ for $x \neq 0$. Let $T \geq 0$ and $x \in \mathbb{R}^{N}$. Since $U$ is a storage function for the closed-loop system formed from (1.1)-(1.2) with $\alpha=\alpha_{U}$, we have

$$
\begin{aligned}
U^{i_{0}}(x) \geq \int_{0}^{T} h\left(y_{x}(s),\right. & \left.\alpha_{x}^{i_{0}}[0](s), 0\right) d s \\
& +\sum_{\tau<T} k\left(\alpha_{U, x}^{i_{0}}\left(\tau^{-}\right), \alpha_{U, x}^{i_{0}}(\tau)\right)+U^{j(T)}\left(y_{x}\left(T, \alpha_{U, x}^{i_{0}}[0], 0\right)\right) .
\end{aligned}
$$

Since $h, k, U$ are nonnegative and $U^{i_{0}}(0)=0$ by our assumptions, substitution of $x=0$ in (4.2) forces

$$
\sum_{\tau<T} k\left(\alpha_{U, 0}^{i_{0}}[0]\left(\tau^{-}\right), \alpha_{U, 0}^{i_{0}}[0](\tau)\right)=0
$$

This implies that $\alpha_{U, 0}^{i_{0}}[0](t)=a^{i_{0}}$ for all $0 \leq t \leq T$. Thus

$$
0 \leq U^{j(T)}\left(y_{0}\left(T, \alpha_{U, 0}^{i_{0}}[0], 0\right)\right)=U^{i_{0}}\left(y_{0}\left(T, \alpha_{U, 0}^{i_{0}}[0], 0\right)\right) \leq U^{i_{0}}(0)=0 .
$$


By the positive definite property of $U^{i_{0}}$, we have $y_{0}\left(T, \alpha_{U, 0}^{i_{0}}[0], 0\right)=0$. Since $T \geq 0$ is arbitrary, we conclude that 0 is an equilibrium point of the system $\dot{y}=f\left(y, a^{i_{0}}, 0\right)$.

Next we want to show that 0 is a globally asymptotically stable equilibrium point for the closed-loop switching system $\Sigma_{s w}$ with $\alpha=\alpha_{U}$. Again, from the storagefunction property of $U=\left(U^{1}, \ldots, U^{r}\right)$ for the system $\Sigma_{s w}$ with $\alpha=\alpha_{U}$, we have

$$
\int_{0}^{T} h\left(y_{x}(s), \alpha_{U, x}^{j}[0](s), 0\right) d s \leq U^{j}(x)<\infty \text { for all } T>0 .
$$

Thus $\lim _{t \rightarrow \infty} h\left(y_{x}(t), \alpha_{U, x}^{j}[0], 0\right)=0$ by Lemma 4.1. By the detectability assumption (v), we have $\lim _{t \rightarrow \infty} y_{x}\left(t, \alpha_{U, x}^{j}[0], 0\right)=0$ as required.

5. An example. We consider in this section an optimal switching problem with one-dimensional state space $\left(x \in \mathbb{R}^{1}\right)$ for which the value function and corresponding control are explicitly computable via a simple geometric construction. There will be two controls: $a \in\{1,2\}$. The switching cost will be symmetric: $k(1,2)=k(2,1)=$ $\beta>0$. For each $a$ value, we will use $a^{\prime}=3-a$ to denote the other control value. The system dynamics will be given by

$$
f(y, 1, b)=-y+b ; \quad f(y, 2, b)=-\mu(y-1)+b,
$$

with output function $h$ taken simply to be the squared state

$$
h(y, a, b)=y^{2} .
$$

We use the specific parameter values

$$
\mu=3, \quad \beta=.4, \quad \gamma=2
$$

throughout.

This example satisfies all of our hypotheses except that we take $B=\mathbb{R}$, which is not compact. Our purpose is to make the SQVI (2.5) more tangible in the context of the example, to show how the optimal strategy $\alpha_{*}$ is determined, and to show how one might establish its optimality. We note that, even apart from the fact that our $B$ is not compact, Theorem 3.9 would not by itself imply that our solution $V^{a}$ of the SQVI is that value of the game (i.e., that $\alpha_{*}$ is optimal); an additional argument to establish the minimality of $V^{a}$ among nonnegative solutions is also necessary. Instead we will outline a direct proof of the optimality of $\alpha_{*}$. Since we are not appealing to any of the theorems above, the fact that the compactness hypothesis on $B$ is not satisfied does not pose a problem. (It would be possible to modify the example so that $B=[-M, M]$ could be used for some sufficiently large $M$. For instance, if the $f(y, a, b)$ were bounded (nonlinear) functions of $y$, this would be possible. However, the linear-affine dynamics of (5.1) are simpler to work with, so we have kept them.)

Our task is to construct the appropriate solution of the SQVI (2.5). With just two control values $a$, the SQVI reduces to the following: for each $a$,

$$
\begin{aligned}
V^{a}(x) & \leq \beta+V^{a^{\prime}}(x) \text { for all } x, \\
H^{a}\left(x, D^{+} V^{a}(x)\right) & \leq 0 \text { for all } x, \\
H^{a}\left(x, D^{-} V^{a}(x)\right) & \geq 0 \text { for those } x \text { with } V^{a}(x)<\beta+V^{a^{\prime}}(x) .
\end{aligned}
$$

Here we have used the standard notation $D^{+} V^{a}(x)$ to refer to the set of all possible slopes $\varphi^{\prime}(x)$ of smooth test functions $\varphi$ for which $V^{a}-\varphi$ has a local maximum at $x$, 
usually called the superdifferential of $V^{a}$ at $x$. Similarly, the subdifferential $D^{-} V^{a}(x)$ denotes the set of all $\varphi^{\prime}(x)$ for smooth test functions $\varphi$ such that $V^{a}(x)-\varphi$ has a local minimum at $x$. (See [6, page 29].) At points $x$ where both $V^{1}$ and $V^{2}$ are differentiable, (5.3)-(5.5) can be expressed more explicitly as

$$
\left|V^{1}(x)-V^{2}(x)\right| \leq \beta
$$

together with the following.

1. If $V^{1}(x)-V^{2}(x)=\beta$, then $\left(V^{1}\right)^{\prime}(x)=\left(V^{2}\right)^{\prime}(x)=: q(x)$ (since $V^{1}-V^{2}$ has a maximum at $x$ ), and

$$
H^{1}(x, q(x)) \leq 0, \quad H^{2}(x, q(x))=0 .
$$

2. If $V^{1}(x)-V^{2}(x)=-\beta$, then, similarly, $\left(V^{1}\right)^{\prime}(x)=\left(V^{2}\right)^{\prime}(x)=: q(x)$ and

$$
H^{1}(x, q(x))=0, \quad H^{2}(x, q(x)) \leq 0 .
$$

3. If $\left|V^{1}(x)-V^{2}(x)\right|<\beta$, then

$$
H^{1}\left(x,\left(V^{1}\right)^{\prime}(x)\right)=0, \quad H^{2}\left(x,\left(V^{2}\right)^{\prime}(x)\right)=0 .
$$

Where one or the other of $V^{a}$ is not differentiable, we must revert to (5.3)-(5.5). However, one of the cases 1-3 above will apply at most $x$.

The two Hamiltonian functions are

$$
\begin{aligned}
& H^{1}(x, p)=p x-x^{2}-\frac{1}{4 \gamma^{2}} p^{2} \\
& H^{2}(x, p)=\mu p(1-x)-x^{2}-\frac{1}{4 \gamma^{2}} p^{2} .
\end{aligned}
$$

These are both instances of the general formula

$$
\begin{aligned}
H(x, p) & =\inf _{b}\left\{-(g(x)+b) \cdot p-x^{2}+\gamma^{2} b^{2}\right\} \\
& =-p g(x)-x^{2}-\frac{1}{4 \gamma^{2}} p^{2} \\
& =(\gamma g(x))^{2}-x^{2}-\left(\frac{1}{2 \gamma} p+\gamma g(x)\right)^{2},
\end{aligned}
$$

where $g(x)=-x$ for $a=1$ and $g(x)=-\mu(x-1)$ for $a=2$. We are interested in $V^{\prime}(x)=p(x)$ solving $H(x, p(x))=0$. Provided $|x|<\gamma|g(x)|$, the equation $H(x, p)=0$ has two distinct real solutions:

$$
-2 \gamma^{2} g(x) \pm 2 \gamma \sqrt{\gamma^{2} g(x)^{2}-x^{2}}
$$

For each $a$, we need to select an appropriate branch $p^{a}(x)$ of the solution to $H^{a}(x, p)=$ 0 . For $a=1$, we take

$$
p^{1}(x)=2 \rho x,
$$

where $\rho=\gamma^{2}-\gamma \sqrt{\gamma^{2}-1}$. Note that, for $x<0, p^{1}(x)$ is the larger of the two solutions of $H^{1}(x, p)=0$, and so

$$
H^{1}(x, p) \leq 0 \text { for all } p \geq p^{1}(x), x<0 .
$$


For $x>0$, however, $p^{1}(x)$ is the smaller of the two solutions, and so

$$
H^{1}(x, p) \leq 0 \text { for all } p \leq p^{1}(x), x>0 .
$$

For $p^{2}(x)$, we note that $H^{2}(x, p)=0$ has no solution for $\frac{6}{7}<x<\frac{6}{5}$ because, with $g(x)=-\mu(x-1),|x|>\gamma|g(x)|$ there. We take

$$
p^{2}(x)= \begin{cases}2 \gamma^{2} \mu(x-1)-2 \gamma \sqrt{\gamma^{2} \mu^{2}(x-1)^{2}-x^{2}} & \text { for } x \geq \frac{6}{5}, \\ 2 \gamma^{2} \mu(x-1)+2 \gamma \sqrt{\gamma^{2} \mu^{2}(x-1)^{2}-x^{2}} & \text { for } x \leq \frac{6}{7}\end{cases}
$$

Let

$$
W^{1}=\rho x^{2}
$$

and

$$
W^{2}(x)=\int p^{2}(x) d x
$$

This determines $W^{2}$ up to two constants, one each for $(-\infty, 6 / 7)$ and $(6 / 5, \infty)$. Those constants are determined uniquely by

$$
W^{2}(x)=\beta+W^{1}(x) \text { for } x=0,3 / 2 .
$$

These are solutions of $H^{a}\left(x, D W^{a}(x)\right)=0$. The desired solutions of the SQVI are given by

$$
V^{2}(x)= \begin{cases}W^{2}(x) & \text { for } x<0 \\ \beta+W^{1}(x) & \text { for } 0 \leq x \leq x_{1} \\ W^{2}(x) & \text { for } x_{1}<x\end{cases}
$$

and

$$
V^{1}(x)= \begin{cases}\beta+W^{2}(x) & \text { for } x \leq x_{2} \\ W^{1}(x) & \text { for } x_{2}<x<x_{3} \\ \beta+W^{2}(x) & \text { for } x_{3} \leq x\end{cases}
$$

where $x_{2}=-1.3175 \ldots, x_{1}=3 / 2, x_{3}=2.55389 \ldots$, values whose significance will emerge below. Graphs are presented in Figure 5.1.

We now outline the verification that $V^{a}$ as defined above do satisfy the SQVI, leaving many of the details to the interested reader. First, consider $0<x<x_{1}$. Here $V^{1 \prime}(x)=p^{1}(x)$ so that $H^{1}\left(x, V^{1 \prime}(x)\right)=0$. Since $V^{2}=\beta+V^{1}$, we also have $V^{2 \prime}(x)=$ $p^{1}(x)$. Case 2 above requires $H^{2}\left(x, p^{1}(x)\right) \leq 0$, which is true up to $x=3 / 2=x_{1}$ but not beyond. For $x_{1}<x<x_{3}$, we have $H^{a}\left(x, V^{a}{ }^{\prime}(x)\right)=H^{a}\left(x, p^{a}(x)\right)=0$ for both $a$, so all of the necessary derivative conditions are satisfied. Note that (5.9) ensures that $V^{2}$ is continuous at $x_{1}$. Moreover, $H^{2}\left(x, p^{1}(x)\right)=0$ at $x=x_{1}$ because $D W^{1}\left(x_{1}\right)=p^{1}\left(x_{1}\right)=p^{2}\left(x_{1}\right)=D W^{2}\left(x_{1}\right)$ there. This means $V^{2}$ is $C^{1}$ at $x_{1}$. We have $V^{1}(x)-\beta<V^{2}(x)<\beta+V^{1}(x)$ for $x_{1}<x<x_{3}$, but at $x_{3}$ we find $V^{1}\left(x_{3}\right)-\beta=$ $V^{2}\left(x_{3}\right)$. (This determines the value of $x_{3}$.) Next consider $x>x_{3}$. Here $V^{2 \prime}=p^{2}(x)$ so that $H^{2}\left(x, V^{2 \prime}(x)\right)=0$. Since $V^{1}(x)=\beta+V^{2}(x), V^{1 \prime}(x)=V^{2 \prime}(x)=p^{2}(x)$ and otherwise case 1 requires only that $H^{1}\left(x, p^{2}(x)\right) \leq 0$, which does hold. Note that the choice of $x_{3}$ makes $V^{1}$ continuous at $x_{3}$, but it is not differentiable there. One finds 


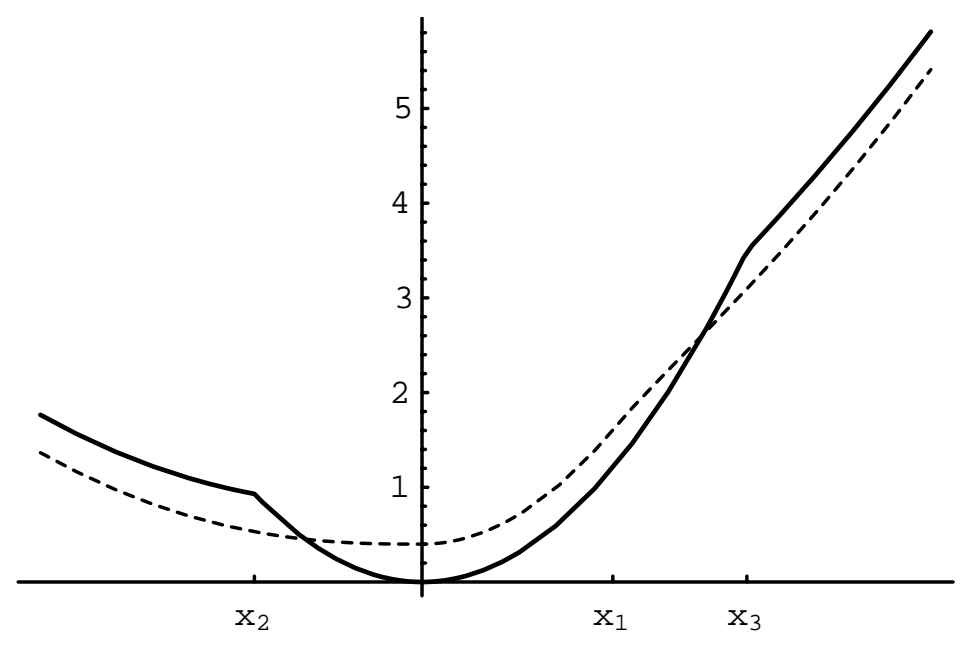

Fig. 5.1. $V^{1}$ (solid) and $V^{2}$ (dashed).

that $D^{-} V^{1}\left(x_{3}\right)=\emptyset$ and $D^{+} V^{1}\left(x_{3}\right)$ is the interval $\left[p^{2}\left(x_{3}\right), p^{1}\left(x_{3}\right)\right]$. By virtue of (5.8), the viscosity solution requirement $H^{1}\left(x_{3}, D^{+} V^{1}\left(x_{3}\right)\right) \leq 0$ is satisfied.

Next consider $x_{2}<x<0$. Here again we have $V^{a}{ }^{\prime}(x)=p^{a}(x)$ so that, for both $a$,

$$
H^{a}\left(x, p^{a}(x)\right)=0 .
$$

We also have $V^{1}(x)-\beta<V^{2}(x)<\beta+V^{1}(x)$, but at $x_{2}$ we find $V^{1}\left(x_{2}\right)-\beta=V^{2}\left(x_{2}\right)$. This determines $x_{2}$ and makes $V^{1}$ continuous at $x_{2}$. For $x<x_{2}$, we have $V^{1 \prime}(x)=$ $V^{2 \prime}(x)=p^{2}(x)$ so that case 1 above requires $H^{2}\left(x, p^{2}(x)\right)=0$ and $H^{1}\left(x, p^{2}(x)\right) \leq 0$, both of which are true for $x<x_{2}$. Note that $V^{1}$ is not differentiable at $x_{2}$. One finds that $D^{-} V^{1}\left(x_{2}\right)=\emptyset$ and $D^{+} V^{1}\left(x_{2}\right)$ is the interval $\left[p^{1}\left(x_{2}\right), p^{2}\left(x_{1}\right)\right]$. By virtue of (5.7), the viscosity solution requirement $H^{1}\left(x_{2}, D^{+} V^{1}\left(x_{2}\right)\right) \leq 0$ is satisfied.

The strategy $\alpha^{*}$ associated with our solution (5.10), (5.11) is easy to describe in state-feedback terms. Define the switching sets

$$
\begin{aligned}
& S_{1}=\left\{x: V^{2}(x)=\beta+V^{1}(x)\right\}=\left[0, x_{1}\right], \\
& S_{2}=\left\{x: V^{1}(x)=\beta+V^{2}(x)\right\}=\left(-\infty, x_{2}\right] \cup\left[x_{3}, \infty\right) .
\end{aligned}
$$

The strategy $\alpha^{*}$ will instantly switch from $a=1$ to $a=2$ whenever $y(t) \in S_{2}$ and will instantly switch from $a=2$ to $a=1$ whenever $y(t) \in S_{1}$. We will prove directly that, in fact, $V_{\gamma}^{a}=V^{a}$ and that our strategy $\alpha^{*}$ is optimal. To be precise, we shall show that, for any $j$ and any strategy $\alpha \in \Gamma$,

$$
V^{j}(y(0)) \leq \sup _{b \in \mathcal{B}} \sup _{T>0}\left\{\int_{0}^{T}\left[h\left(y_{x}(s), \alpha_{x}^{j}[b](s), b(s)\right)-\gamma^{2}|b(s)|^{2}\right] d s+\sum_{\tau_{i} \leq T} k\left(a_{i-i}, a_{i}\right)\right\} .
$$

Moreover, for our strategy $\alpha^{*},(5.12)$ will be an equality for all $x, j$. The key to this is the existence of a particular "worst case" disturbance, as claimed by the following proposition. (This proposition is intended only in the context of the particular example and the parameter values described above.) 
Proposition 5.1. For any $x \in \mathbb{R}^{N}, j \in\{1,2\}$, and strategy $\alpha \in \Gamma$, there exists a disturbance $b^{*}=b_{\alpha_{x}^{j}}^{*} \in \mathcal{B}$ with the property that

$$
b^{*}(t)=\frac{1}{2 \gamma^{2}}\left(V^{\left.\alpha_{x}^{j}\left[b^{*}\right]\right)(t)}\right)^{\prime}\left(y_{x}\left(t, \alpha_{x}^{j}[b], b\right)\right)
$$

holds for all but finitely many $t$ in every interval $[0, T]$.

A proof can be based on the obvious construction. Given $\alpha \in \Gamma$, an initial control $j$, and an initial point $x \in \mathbb{R}^{N}$, consider the solution of

$$
\dot{y}=f\left(y, j, \frac{1}{\gamma^{2}}\left(V^{j}\right)^{\prime}(y)\right) ; \quad y(0)=x .
$$

We simply take $b^{*}(t)=\left(V^{j}\right)^{\prime}(y(t))$ up until the first time $\tau_{1}$ that the policy $\alpha_{x}^{j}\left[b^{*}\right]$ calls for a switch from $a=j$ to $a=j^{\prime}$. For $t>\tau_{1}$, we continue by solving

$$
\dot{y}=f\left(y, j^{\prime}, \frac{1}{\gamma^{2}}\left(V^{j^{\prime}}\right)^{\prime}(y)\right)
$$

with initial value $y\left(\tau_{1}\right)$ as already determined. We take $b^{*}(t)=\left(V^{j^{\prime}}\right)^{\prime}(y(t))$ for $\tau_{1}<t$ up until the next time $\tau_{2}$ that $\alpha_{x}^{j}\left[b^{*}\right]$ calls for a switch from $a=j^{\prime}$ to $a=j$. We continue this construction iteratively.

A number of observations are needed to justify the construction. One is the existence of a unique solution to (5.13). For $j=2$, the right side is $C^{1}$, so the solution is uniquely determined. For $j=1$, the right side has discontinuities at $x_{2}$ and $x_{3}$, but, since $f\left(x, 1, \frac{1}{\gamma^{2}}\left(V^{1}\right)^{\prime}(x)\right)$ does not change sign across the discontinuities, the solution is uniquely determined. Graphs of $f\left(y, a, \frac{1}{\gamma^{2}}\left(V^{a}\right)^{\prime}(y)\right)$ are provided in Figures 5.2 and 5.3 below. (We comment that, although the graphs appear piecewise linear, they are not. Figure 5.2 is linear only for $0<x<x_{1}$, and Figure 5.3 is linear only for $x_{2}<x<x_{3}$, as inspection of the formulas shows.) One can check that $y \dot{y}<0$ for sufficiently large $|y|$, which implies that solutions of (5.13) are defined for all $t \geq 0$. Observe also for $j=1$ that, for any solution of (5.13), there is at most one value of $t$ for which $y(t)$ is at one of the discontinuities of $\left(V^{1}\right)^{\prime}$ and for which there is any ambiguity in the specification $b^{*}(t)=\left(V^{j}\right)^{\prime}(y(t))$.

The other concern is that the sequence $\tau_{i}$ of switching times generated by our construction might have a finite accumulation point: $\lim \tau_{i}=s<\infty$. Our hypotheses on the strategy $\alpha$ disallow this, however, for the following reason. If it were the case that $\lim \tau_{i}=s<\infty$, then extend our definition of $b^{*}$ in any way to $t \geq s$, say, $b^{*}(t)=0$. By hypothesis, $\alpha_{x}^{j}\left[b^{*}\right]$ is an admissible control in $\mathcal{A}$, which means, in particular, that its switching times $\tau_{i}$ do not have a finite accumulation point. However, extension of $b^{*}$ for $t>s$ does not alter the switching times $\tau_{i}<s$ by the nonanticipating property of $\alpha$. This would mean that $\alpha\left[b^{*}\right]$ does have an infinite number of switching times $\tau_{i}<s$, which is a contradiction. Finally, by our comments above, on each interval $\left[\tau_{i}, \tau_{i+1}\right]$, there is at most a single $t$ value at which $b^{*}(t)=\left(V^{\alpha_{x}^{j}\left[b^{*}\right]}\right)^{\prime}(y(t))$ is ambiguous. Thus there are at most a finite number of such $t$ in any $[0, T]$.

Consider now any strategy $\alpha \in \Gamma$, initial position $x=y(0)$, and control setting $j$, and let $b^{*}(t)$ be the disturbance described in the proposition. We will let $a_{i}=\alpha_{x}^{j}\left[b^{*}\right](t)$ denote the control settings on the intervals $\left[\tau_{i}, \tau_{i+1}\right]$ between consecutive switching times. In particular, $a_{0}=j$. On each interval $\left[\tau_{i}, \tau_{i+1}\right],(5.4)$ and the fact that $b^{*}(t)$ achieves the infimum in (5.6) for $x=y(t)$ and $p=\left(V^{a_{i}}\right)^{\prime}(x)$ imply that (for all but 


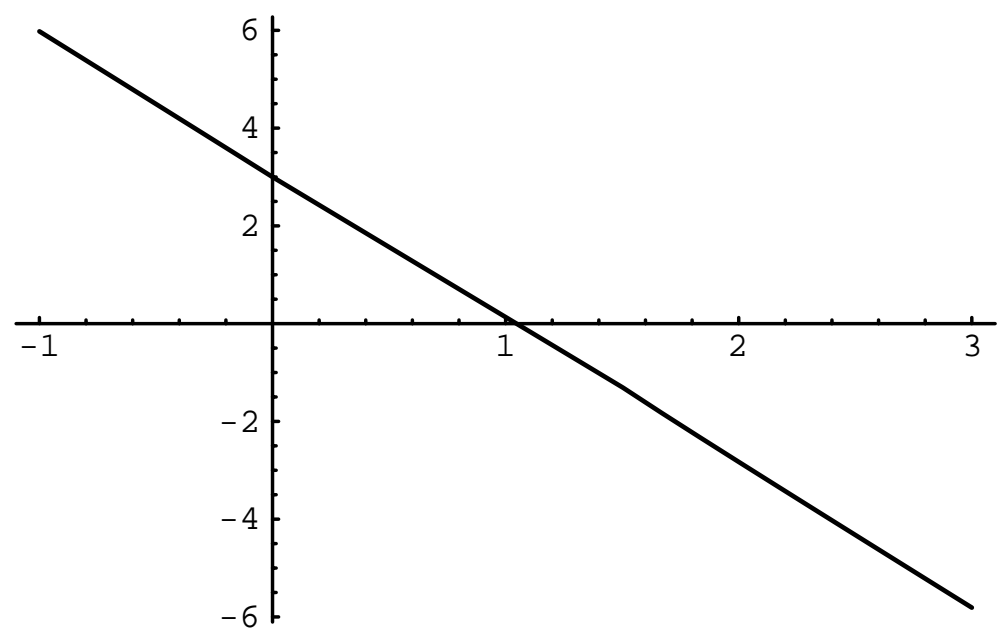

FIG. 5.2. Plot of $f\left(x, 2, \frac{1}{2 \gamma^{2}} V^{2 \prime}(x)\right)$.

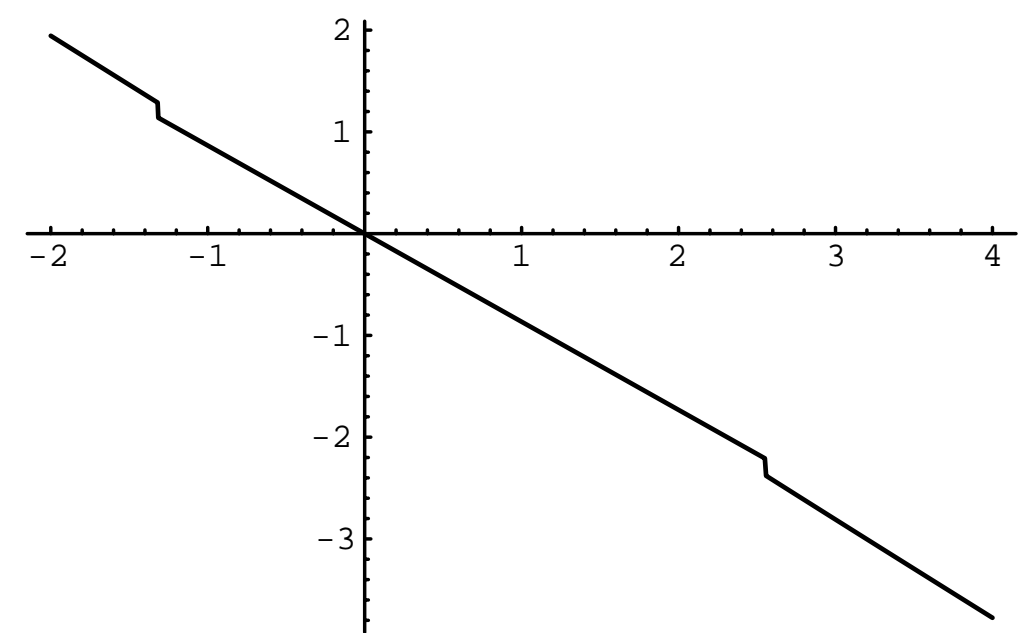

FIG. 5.3. Plot of $f\left(x, 1, \frac{1}{2 \gamma^{2}} V^{1 \prime}(x)\right)$.

one $t$ )

$$
\frac{d}{d t} V^{a_{i}}(y(t)) \geq \gamma^{2} b^{*}(t)^{2}-h\left(y(t), a_{i}, b^{*}(t)\right) .
$$

Thus, for any $\tau_{i}<t \leq \tau_{i+1}$, we have

$$
V^{a_{i}}(y(t))-V^{a_{i}}\left(y\left(\tau_{i}\right)\right) \geq \int_{\tau_{i}}^{t}\left[\gamma^{2}\left|b^{*}(s)\right|^{2}-h(s)\right] d s .
$$

Across a switching time $\tau_{i}$, we have from (5.3)

$$
V^{a_{i}}\left(y\left(\tau_{i}\right)\right)-V^{a_{i-1}}\left(y\left(\tau_{i}\right)\right) \geq-\beta=-k\left(a_{i-1}, a_{i}\right)
$$


Adding these inequalities over $\tau_{i} \leq T$, we see that

$$
V^{\alpha\left[b^{*}\right](T)}(y(T))-V^{\alpha\left[b^{*}\right](0)}(y(0)) \geq-\left\{\int_{0}^{T}\left[h(s)-\gamma^{2}\left|b^{*}(s)\right|^{2}\right] d s+\sum_{\tau_{i} \leq T} k\left(a_{i-1}, a_{i}\right)\right\} .
$$

A rearrangement of this gives

$$
V^{\alpha\left[b^{*}\right](T)}(y(T))+\left\{\int_{0}^{T}\left[h(s)-\gamma^{2}\left|b^{*}(s)\right|^{2}\right] d s+\sum_{\tau_{i} \leq T} k\left(a_{i-1}, a_{i}\right)\right\} \geq V^{\alpha\left[b^{*}\right](0)}(y(0)) .
$$

When we consider $\alpha^{*}$ specifically, we recognize that

$$
H^{a_{i}}\left(y(t),\left(V^{a_{i}}\right)^{\prime}(y(t))\right)=0
$$

for $t$ between two $\tau_{i}$ 's, and at $\tau_{i}$ we have

$$
V^{a_{i+1}}\left(y\left(\tau_{i}\right)\right)-V^{a_{i}}\left(y\left(\tau_{i}\right)\right)=-\beta=-k\left(a_{i+1}, a_{i}\right) .
$$

This means that (5.14) is an equality for $\alpha^{*}$ specifically.

To finish our optimality argument, we will show that, for $\alpha$, a general strategy, initial condition $\left(x, a^{j}\right)$, and associated disturbance $b^{*}=b_{\alpha_{x}^{j}}^{*}$ as above, as $T \rightarrow \infty$ we must have either $y(T) \rightarrow 0$ and $\alpha\left[b^{*}\right](T) \rightarrow 1$, or else

$$
\int_{0}^{T}\left[h(s)-\gamma^{2}\left|b^{*}(s)\right|^{2}\right] d s+\sum_{\tau_{i} \leq T} k\left(a_{i-1}, a_{i}\right) \rightarrow+\infty .
$$

In the case of $\alpha=\alpha^{*}$ specifically, we will have the former possibility. Since $V^{1}(0)=0$ and is continuous, these facts imply (5.12) as claimed. The verification of these asserted limiting properties for the case of general $\alpha$ depends on some particular inequalities for $\left(V^{a}\right)^{\prime}(x)$ as determined by (5.11), (5.10). First, we assert that, for both $a$ values,

$$
h\left(y(t), a, b^{*}(t)\right)-\gamma^{2}\left|b^{*}(t)\right|^{2}=|y(t)|^{2}-\frac{1}{4 \gamma^{2}}\left[\left(V^{a}\right)^{\prime}(y(t))\right]^{2}>0 \text { for } x \neq 0 .
$$

Moreover, $|x|^{2}-\frac{1}{4 \gamma^{2}}\left[\left(V^{a}\right)^{\prime}(x)^{2}\right.$ has a positive lower bound on $\{x:|x| \geq \epsilon\}$ for each $\epsilon>0$. Instead of what would be a very tedious algebraic demonstration of this, we simply offer the graphical demonstration in Figure 5.4. We have plotted $b^{*}=\frac{1}{2 \gamma}\left(V^{a}\right)^{\prime}(x)$ (solid lines) and $q=x$ (dashed lines) as functions of $x$. The validity of (5.16) is apparent.

The other fact we need is that, for $a=2$ and the corresponding disturbance $b^{*}(t)$, the state-dynamics do not have an equilibrium at 0 . This is easy to see because at $x=0$ we have $b^{*}=\frac{1}{2 \gamma^{2}}\left(V^{2}\right)^{\prime}(0)=0$, but $f\left(0,2, b^{*}\right)=-\mu+b^{*}$. A graph of $f\left(x, 2, b^{*}\right)=-\mu(x-1)+\frac{1}{2 \gamma^{2}}\left(V^{2}\right)^{\prime}(x)$ is provided in Figure 5.2, where we see the unique equilibrium just beyond $x=1$.

In the case of $a=1$, however, $\dot{x}=f\left(x, 1, \frac{1}{2 \gamma^{2}}\left(V^{1}\right)^{\prime}(x)\right)$ has a unique globally asymptotically stable equilibrium at $x=0$, as is evident in Figure 5.3. 

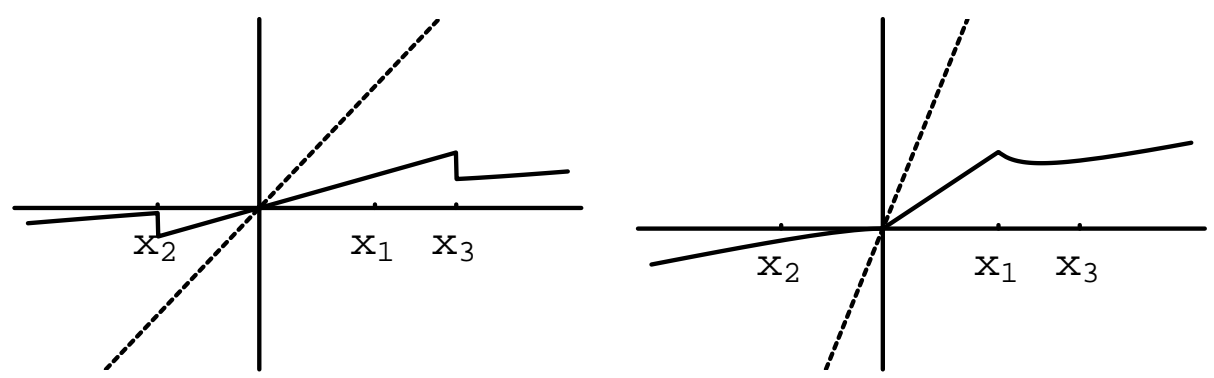

FIG. 5.4. Graphical verification of (5.16) for $V^{1 \prime}$ (left) and $V^{2 \prime}$ (right).

We turn then to the verification of the assertion of (5.15) or its alternative: assuming (5.15) to be false, we claim that $y(T) \rightarrow 0$ and $\alpha\left[b^{*}\right](T) \rightarrow 1$. By the nonnegativity from (5.16), we must have both

$$
\sum_{\tau_{i}<\infty} k\left(a_{i-1}, a_{i}\right)<\infty \text { and } \int_{0}^{\infty}\left[h(y(s))-\gamma^{2}\left|b^{*}(s)\right|^{2}\right] d s<\infty .
$$

The first of these implies that there are only a finite number of switches; $\alpha\left[b^{*}\right](t)=a^{i^{*}}$ is constant from some time on. It is not possible that $i^{*}=2$ because, in that case, $y(t)$ would be converging to the positive equilibrium of Figure 5.2, which implies by (5.16) that, as $t \rightarrow \infty$,

$$
h\left(y(t), a^{i^{*}}, b^{*}(t)\right)-\gamma^{*}\left|b^{*}(t)\right|^{2} \rightarrow C>0 .
$$

This contradicts the second part of (5.17). Therefore, $i^{*}=1$, which shows that $\alpha\left[b^{*}\right](T) \rightarrow 1$. However, since $\alpha\left[b^{*}\right](t)=1$ from some point on, the stability illustrated in Figure 5.3 means that $y(t) \rightarrow 0$ as claimed. This completes our verification of the optimality of the strategy $\alpha^{*}$.

\section{REFERENCES}

[1] J. A. Ball And J. Chudoung, Comparison theorems for viscosity solutions of systems of quasivariational inequalities with applications to switching-cost control problems, J. Math. Anal. Appl., 251 (2000), pp. 40-64.

[2] J. A. Ball, J. Chudoung, And M. V. DAy, Robust optimal stopping-time control for nonlinear systems, Appl. Math. Optim., 29 (2002).

[3] J. A. BAll, M. V. DAY, T. YU, AND P. KaChroo, Robust L $L_{2}$-gain control for nonlinear systems with projection dynamics and input constraints: An example from traffic control, Automatica J. IFAC, 35 (1999), pp. 429-444.

[4] J. A. BAll, M. V. DAY, AND P. KACHROo, Robust feedback control of a single server queueing system, Math. Control Signals Systems, 12 (1999), pp. 307-345.

[5] J. A. BAll AND J. W. Helton, Viscosity solutions of Hamilton-Jacobi equations arising in nonlinear $H_{\infty}$ control, J. Math. Systems Estim. Control, 6 (1996), pp. 1-22.

[6] M. Bardi and I. Cappuzzo-Dolcetta, Optimal Control and Viscosity Solutions of HamiltonJacobi-Bellman Equations, Birkhäuser Boston, Boston, 1997.

[7] E. N. Barron, R. Jensen, and J. L. Menaldi, Optimal control and differential games with measures, Nonlinear Anal., 21 (1993), pp. 241-268.

[8] T. Başar and P. Bernhard, $H^{\infty}$-Optimal Control and Related Minimax Design Problems: A Dynamic Game Approach, 2nd ed., Birkhäuser Boston, Boston, 1995.

[9] A. Bensoussan and J. L. Lions, Applications of Variational Inequalities to Stochastic Control, North-Holland, New York, 1982.

[10] M. S. Branicky, V. S. Borkar, and S. K. Mitter, A unified framework for hybrid control: Model and optimal control theory, IEEE Trans. Automat. Control, 43 (1998), pp. 31-45. 
[11] M.S. Branicky, Multiple Lyapunov functions and other analysis tools for switched and hybrid systems, IEEE Trans. Automat. Control, 43 (1998), pp. 475-482.

[12] M. G. Crandall, H. Ishit, and P.-L. Lions, User's guide to viscosity solutions of second order partial differential equations, Bull. Amer. Math. Soc. (N.S.), 27 (1992), pp. 1-67.

[13] H. Frankowska and M. Quincampoix, Dissipative control systems and disturbance attenuation for nonlinear $H^{\infty}$ problems, Appl. Math. Optim., 40 (1999), pp. 163-181.

[14] J. W. Helton and M. R. James, Extending $H_{\infty}$ Control to Nonlinear Systems: Control of Nonlinear Systems to Achieve Performance Objectives, SIAM, Philadelphia, 1999.

[15] M. R. JAmes, A partial differential inequality for dissipative nonlinear systems, Systems Control Lett., 21 (1993), pp. 315-320.

[16] D. Liberzon And A. S. Morse, Basic problems in stability and design of switched systems, Control Systems, 19 (1999), pp. 59-70.

[17] M. Motta and F. Rampazzo, Space-time trajectories of nonlinear systems driven by ordinary and impulsive control, Differential Integral Equations, 8 (1995), pp. 269-288.

[18] M. Motta And F. Rampazzo, Dynamic programming for nonlinear systems driven by ordinary and impulsive controls, SIAM J. Control Optim., 34 (1996), pp. 199-225.

[19] S. Sastry And M. Bodson, Adaptive Systems: Stability, Convergence and Robustness, Prentice-Hall, Englewood Cliffs, NJ, 1989.

[20] A. VAn Der Schaft, $L_{2}$-gain and Passivity Techniques in Nonlinear Control, Springer-Verlag, New York, 1996.

[21] P. Soravia, $H_{\infty}$ control of nonlinear systems: Differential games and viscosity solutions, SIAM J. Control Optim., 34 (1996), pp. 1071-1097.

[22] P. Soravia, Equivalence between nonlinear $H_{\infty}$ control problems and existence of viscosity solutions of Hamilton-Jacobi-Isaacs equations, Appl. Math. Optim., 39 (1999), pp. 17-32.

[23] J. YONG, A zero-sum differential game in a finite duration with switching strategies, SIAM J. Control Optim., 28 (1990), pp. 1234-1250.

[24] J. YonG, Differential games with switching strategies, J. Math. Anal. Appl., 145 (1990), pp. $455-469$. 